\title{
Timing performance of a multi-pad PICOSEC-Micromegas detector prototype
}

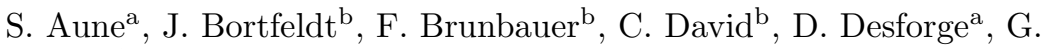 \\ Fanourakis $^{f}$, M. Gallinaro ${ }^{\mathrm{h}}$, F. García ${ }^{\mathrm{l}}$, I. Giomataris ${ }^{\mathrm{a}}$, T. Gustavsson ${ }^{\mathrm{j}}$, F.J.

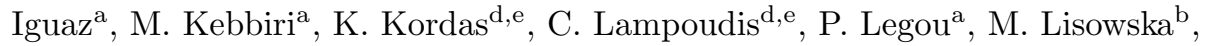 \\ J. Liu ${ }^{\mathrm{c}}$, M. Lupberger ${ }^{\mathrm{b}, 1}$, O. Maillard ${ }^{\mathrm{a}}$, I. Maniatis ${ }^{\mathrm{d}, \mathrm{e}}$, I. Manthos ${ }^{\mathrm{d}, \mathrm{e}}$, H. \\ Müller $^{\mathrm{b}}$, E. Oliveri ${ }^{\mathrm{b}}$, T. Papaevangelou ${ }^{\mathrm{a}}$, K. Paraschou ${ }^{\mathrm{d}}$, M. Pomorski $^{\mathrm{k}}$, B. \\ $\mathrm{Qi}^{\mathrm{c}}$, F. Resnati ${ }^{\mathrm{b}}$, L. Ropelewski $^{\mathrm{b}}$, D. Sampsonidis ${ }^{\mathrm{d}, \mathrm{e}}$, L. Scharenberg $^{\mathrm{b}}, \mathrm{T}$.

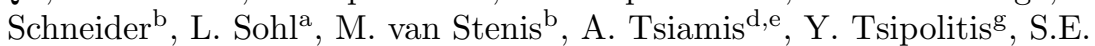

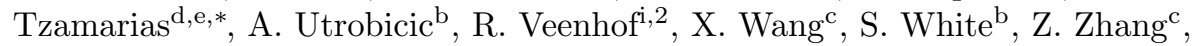 \\ Y. Zhou ${ }^{\mathrm{c}}$ \\ ${ }^{a} I R F U, C E A$, Universit'e Paris-Saclay, F-91191 Gif-sur-Yvette, France \\ ${ }^{b}$ European Organization for Nuclear Research (CERN), CH-1211 Geneve 23, Switzerland \\ ${ }^{c}$ State Key Laboratory of Particle Detection and Electronics, University of Science and \\ Technology of China, Hefei CN-230026, China \\ ${ }^{d}$ Department of Physics, Aristotle University of Thessaloniki, University Campus, \\ GR-54124, Thessaloniki, Greece. \\ ${ }^{e}$ Center for Interdisciplinary Research and Innovation (CIRI-AUTH), Thessaloniki 57001, \\ Greece. \\ Institute of Nuclear and Particle Physics, NCSR Demokritos, GR-15341 Agia Paraskevi, \\ Attiki, Greece \\ ${ }^{g}$ National Technical University of Athens, Athens, Greece \\ ${ }^{h}$ Laboratório de Instrumentacão e Física Experimental de Partículas, Lisbon, Portugal \\ ${ }^{i}$ RD51 collaboration, European Organization for Nuclear Research (CERN), CH-1211 \\ Geneve 23, Switzerland \\ ${ }^{j}$ LIDYL, CEA, CNRS, Universit Paris-Saclay, F-91191 Gif-sur-Yvette, France \\ ${ }^{k}$ CEA-LIST, Diamond Sensors Laboratory, CEA Saclay, F-91191 Gif-sur-Yvette, France \\ ${ }^{l}$ Helsinki Institute of Physics, University of Helsinki, FI-00014 Helsinki, Finland
}

\begin{abstract}
The multi-pad PICOSEC-Micromegas is an improved detector prototype with a segmented anode, consisting of 19 hexagonal pads. Detailed studies are performed with data collected in a muon beam over four representative pads. We demonstrate that such a device, scalable to a larger area, provides excellent time resolution and detection efficiency. As expected from earlier single-cell device studies, we measure a time resolution of approximately 25 picoseconds for charged particles hitting near the anode pad centers, and up to 30 picoseconds

\footnotetext{
* Corresponding author

Email address: tzamarias@auth.gr (S.E. Tzamarias)

${ }^{1}$ Now at University of Bonn, D-53115 Bonn, Germany.

${ }^{2}$ Also at National Research Nuclear University MEPhI, Kashirskoe Highway 31, Moscow, Russia; and Department of Physics, Uludağ University, 16059 Bursa,Turkey.
} 
at the pad edges. Here, we study in detail the effect of drift gap thickness non-uniformity on the timing performance and evaluate impact position based corrections to obtain a uniform timing response over the full detector coverage.

Keywords: gaseous detectors, Micromegas, multi-pad, time resolution

\section{Introduction}

The ability to precisely measure the production time of minimum ionizing particles (MIPs) enables new capabilities to the current experiments in High Energy and Nuclear Physics and extend their physics reach. As the scale and level of performance of the timing components of current experiments continues to advance, so do the roles for timing of physics objects and the technologies available for sensors proliferate. Aside from the traditional role of time-of-flight for charged particle identification [1], the LHC experiments are implementing charged particle timing to mitigate pileup-induced backgrounds for the coming higher luminosity era (HL-LHC). At the LHC, precision timing is also being implemented in components of the calorimetry for the same purpose [2].

Aside from the LHC, timing capability is also considered at new facilities and in underground experiments - the latter principally for discrimination between scintillation and Cherenkov photons 3. While the LHC upgrades have focused on silicon-based sensors with internal gain for the approved projects, $\mathrm{R} \& \mathrm{D}$ continues on timing with silicon without internal gain [4] and devices with intermediate gain [5]. Nevertheless, it has become clear that there is a need to keep the detector capacitance small for noise considerations, which can be achieved by increasing the detector granularity. However, the consequent cost per unit area of such highly granular systems are likely to make siliconbased detectors unattractive for applications requiring more than a few square meters of coverage.

Our collaboration demonstrated that charged particle time-of-arrival resolution of better than 25 picoseconds and single-photon timing precision of roughly 50 picoseconds can be achieved [6, 7] with an implementation of single cell Micromegas detectors coupled to a few-mm thick UV-transparent Cherenkov radiator (PICOSEC-Micromegas). Here, we report on a new PICOSEC-Micromegas device with 19 hexagonal anode pads (a multi-pad PICOSEC-Micromegas prototype) and we evaluate in detail the timing performance for muons impinging onto four representative pads. In the multi-pad device discussed here, we study in detail the effect of drift gap thickness non-uniformity on the timing performance and devise corrections to obtain a uniform timing response over the full detector coverage. Our work highlights the critical role of tight mechanical tolerances on the depth of the drift/preamplification stage.

After describing the multi-pad PICOSEC-Micromegas prototype (Section2), the experimental setup used for collecting calibration data in a $150 \mathrm{GeV}$ muon beam and the processing of the detector signals (Section 3), we report on the 

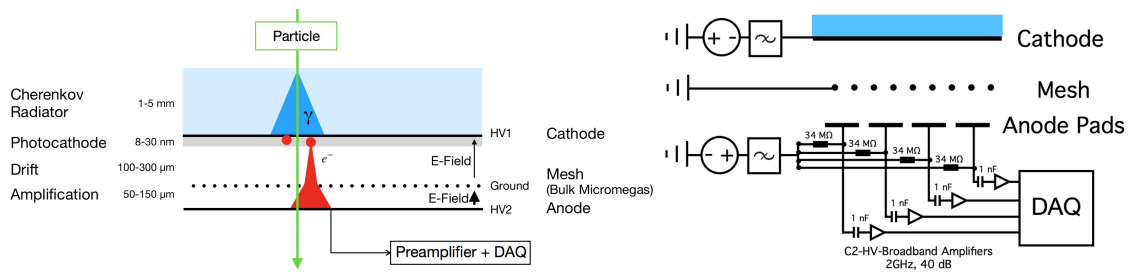

Figure 1: (left) Sketch of the PICOSEC-Micromegas working principle. (right) Electrical diagram of the detector power supply and signal read-out. The signal of each anode pad is individually amplified with a CIVIDEC C2-HV broadband amplifier.

method used for a precise detector alignment and on the findings indicating the non-uniformity of the drift gap thickness (Section 4). In Section 5 we investigate the effect of the above non-uniformity on the timing characteristics of each of the instrumented pads and we evaluate corrections that restore uniformity and improve the time resolution. In Section 6 we report on a method to combine timing and charge information from neighboring pads to estimate the arrival times of MIPs that impact the detector close to pad edges. Section 7 contains concluding remarks.

\section{The multi-pad PICOSEC-Micromegas detector}

The main idea of the PICOSEC-Micromegas concept is to suppress the inevitable time jitter of the ionisation in a Micromegas [8, due to different ionisation cluster positions. Figure 1 illustrates the PICOSEC-Micromegas detector concept. A Cherenkov radiator and a photocathode are placed in front of the gaseous volume. The passage of a charged particle through the Cherenkov radiator produces UV photons, which are then absorbed in the photocathode and primary electrons (photoelectrons) are created on the bottom surface of the photocathode. These electrons are subsequently preamplified in a first and then amplified in a second high-field stage, inducing a signal on the read-out plane.

The drift region thickness in the PICOSEC-Micromegas is reduced to the same order as the amplification gap. It is moreover operated with an electric field similar to that of the amplification gap. In this field configuration, a preamplification of the electrons happens in the drift gap improving the time resolution as it reduces the drift time of the primary electrons [6, 9].

A PICOSEC-Micromegas detector with an active area of $3.5 \mathrm{~cm}$ diameter has been developed with a $M g F_{2}$ Cherenkov radiator, $5 \mathrm{~cm}$ in diameter. A layer of $C s I$, with a thickness of $18 \mathrm{~nm}$ has been used as UV semi-transparent photocathode. A thin $C r$ layer, with a thickness of about $3 \mathrm{~nm}$, has been used as conductive interface layer between radiator and CsI. The UV transmission of the $M g F_{2}$ radiator at $160 \mathrm{~nm}$ is about $80 \%$. An additional $50 \%$ of transmission decrease has been measured at the same wavelength when the $\mathrm{Cr}$ layer is present. 

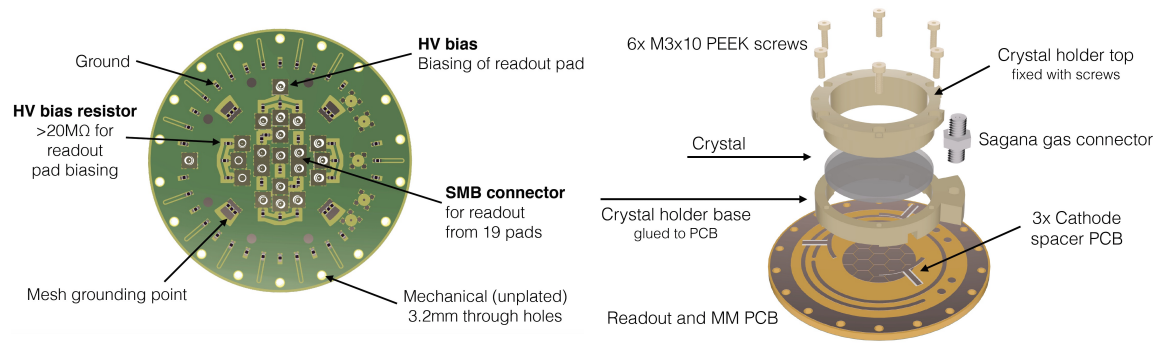

Figure 2: (left) Technical drawing of the PCB. (right) Technical sketch of the mechanics that holds the Cherenkov crystal with photocathode in place.

A bulk Micromegas [10] with $184 \mu \mathrm{m}$ drift gap and $128 \mu \mathrm{m}$ amplification gap is used for this detector with the mesh directly connected to ground on the PCB. A calendered mesh with $18 \mu \mathrm{m}$ wire diameter, $45 \mu \mathrm{m}$ opening and about $30 \mu \mathrm{m}$ thickness is used.

This is the first PICOSEC-Micromegas detector with a segmented readout anode divided into 19 hexagonal pads with a diameter of $1 \mathrm{~cm}$ each. The adjacent edges of neighboring pads are separated by $200 \mu \mathrm{m}$ gap. The left sketch in Fig. 2 shows a technical drawing of the read-out PCB and the right sketch in the same figure shows the mounting mechanism of the Cherenkov radiator on top of the PCB. Only the seven inner hexagons are printed in full size as the detector boundary curtails the outer hexagons. The left photograph in Fig. 3 shows a top view picture of the detector during assembly in the clean-room. The pad structure with the full pads in the centre and the cut pads on the border are visible.

The PCB with the Micromegas is mounted on one flange that seals the chamber hermetically. Over- and under-pressure operation as well as mechanical stress during assembly have therefore a direct impact on the PCB flatness. As the PCB is sealing the detector, the signal for each pad is individually routed to the back of the PCB by an individual SMB (SubMiniature version B) connector. The high voltage is instead supplied through one common connector for all pads. The high voltage is decoupled to each pad connector by a resistor $(>20 \mathrm{M} \Omega)$ to allow a separate read-out of some pads while powering the whole detector.

Unlike the previous PICOSEC-Micromegas [6] that had a window in the outer gas vessel, this detector is completely sealed and has no windows. In order to calibrate the detector, a UV-LED is mounted inside the chamber with the bias voltage fed through the PCB.

\section{Experimental setup and waveform processing}

The time response of the multi-pad PICOSEC-Micromegas to $150 \mathrm{GeV}$ muons was measured at the CERN SPS H4 secondary beamline. A similar experimental setup as the one used in previous measurements [6] allows the 


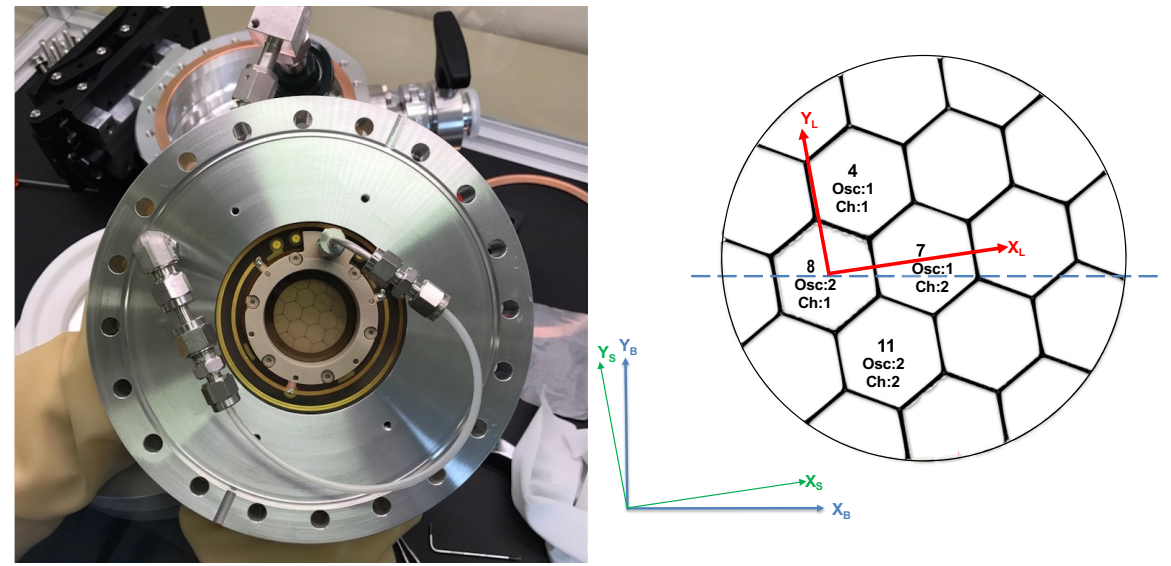

Figure 3: (left) Photograph of the multi-pad chamber during assembly in the clean room. The hexagonal pad structure of the readout is visible in the centre. (right) A schematic diagram of the anode segmentation. Notice that there is a gap between adjacent pad edges, represented by the thick black lines. The pads No. 4, 7, 8 and 11 are fully instrumented and their signals are digitized by the oscilloscope channels as indicated. The red axes, labelled as $X_{L}$ and $Y_{L}$, represent the local coordinate frame, while the blue axes, labelled as $X_{B}$ and $Y_{B}$, represent the global tracking coordinate frame (or else beam-frame). The green axes, labelled as $X_{S}$ and $Y_{S}$, represent the symmetry frame, which is used in the alignment procedure as described in the text.

characterization of the detector prototype, situated as shown in Fig. 4. Two trigger scintillators of $5 \times 5 \mathrm{~mm}^{2}$ operate in anti-coincidence with a veto scintillator whose aperture (hole) matches the same area (small area trigger). This trigger configuration efficiently selects muons that do not undergo scattering and suppresses triggers from particle showers. Another set of trigger scintillators is available covering a $5 \times 5 \mathrm{~cm}^{2}$ area (large area trigger). This trigger is selected to study several pads in parallel. One PHOTEK MCP - PMT, model PMT240/Q/BI/NG77 11, operated at $-4800 \mathrm{~V}$, provides the time reference; its entrance window (9 $\mathrm{mm}$ Fused Silica) is placed perpendicular to the beam and serves as a Cherenkov radiator. In a previous work [12, we determined that the MCP provides a reference time with an accuracy of $\sim 7 \mathrm{ps}$ in the inner $11 \mathrm{~mm}$ diameter. A telescope of three tracking GEM detectors with two-dimensional strip readout is used to reconstruct the trajectory of each muon with a combinatorial Kalman filter based algorithm, and to determine its impact position at the PICOSEC-Micromegas photocathode with an accuracy of about $50 \mu \mathrm{m}$.

The signals of the four instrumented PICOSEC-Micromegas pads go through CIVIDEC 13 preamplifiers before being digitized and recorded (together with the MCP signal) by two LECROY WR8104 oscilloscopes 14 operated at 1.0 $\mathrm{GHz}$ analogue bandwidth and at a sampling rate of 10 GSamples/s. Specifically, the signals from pads No. 4 and 7 are digitized by channels Ch:1 and Ch:2 of the first oscilloscope (Osc:1), while the signals from pads No. 8 and 11 are digitized by $\mathrm{Ch}: 1$ and $\mathrm{Ch}: 2$ of the second oscilloscope (Osc:2), respectively 


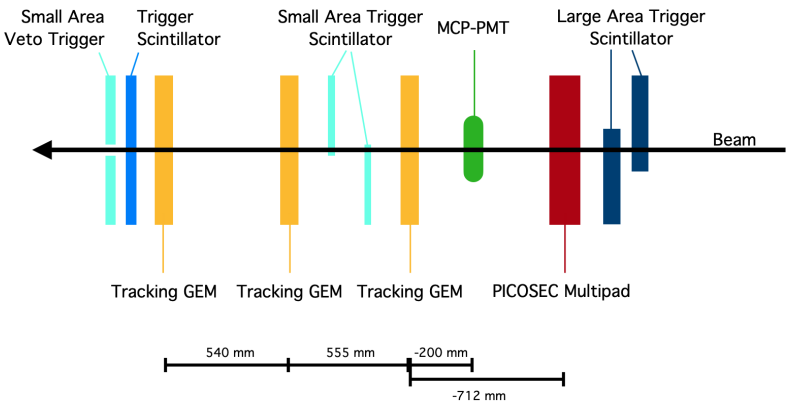

Figure 4: Layout of the experimental setup (not to scale) during the beam tests. The incoming beam enters from the right side of the figure; events are triggered by either the coincidence of two $5 \times 5 \mathrm{~mm}^{2}$ scintillators in anti-coincidence with a "veto" scintillator (small area trigger) or by the coincidence of two $5 \times 5 \mathrm{~cm}^{2}$ scintillators (large area trigger). Three GEM detectors provide tracking information of the incoming charged particles, and the MCPPMTs provide the timing reference for the PICOSEC-Micromegas measurements. Details are given in the text.

(Fig. 3). The MCP signal, after being split by a $50 \mathrm{Ohm}$ splitter, is digitized by the third channel of both oscilloscopes, while the tracking (GEM detector) data are recorded simultaneously in an APV25 based SRS DAQ [15. The SRS DAQ system of the tracking detectors is triggered by the scintillator triggers, definning the acceptance area of the beam particles. To ensure event alignment in the two DAQ systems, the internal SRS event number is sent as a bit stream to the fourth channels of both oscilloscopes and is used to trigger the acquisition of the PICOSEC-Micromegas waveforms.

The PICOSEC-Micromegas detector was operated with the COMPASs $3^{3}$ gas at atmospheric pressure and at different anode and drift voltages, with the detector gain exceeding $10^{5}$. The data used in this work are collected during a long run, when more than 500,000 triggers were collected with the detector operated under stable condition, with $300 \mathrm{~V}$ anode and $450 \mathrm{~V}$ drift voltages.

The digitized PICOSEC-Micromegas waveforms were processed according to the following steps as in [6]: first by determining the baseline offset and noise level using the $75 \mathrm{~ns}$ precursor of the pulse, then measuring the "electron-peak" amplitude $\left(V_{\max }\right)$ as the difference between the maximum of the waveform and the baseline. Finally a logistic function is fitted to the leading edge of the electron-peak to determine the CF20 (20\% constant fraction) time as the time when the fitted function equals $20 \%$ of $V_{\max }$.

For the MCP digitized waveforms a simpler approach is followed, as these signals are very fast and almost noise-free: after the calculation of the pulse

\footnotetext{
${ }^{3}$ The term "COMPASS gas" refers to the mixture $80 \% \mathrm{Ne}, 10 \% \mathrm{C}_{2} \mathrm{H}_{6}, 10 \% \mathrm{CF}_{4}$, as used by the COMPASS Collaboration.
} 
baseline and amplitude, a linear interpolation between two points around CF20 is used to extract the temporal position of the signal.

The "Signal Arrival Time" (SAT) is defined as the difference between the CF20 time of the PICOSEC-Micromegas pad and that of the MCP waveform, both digitized by the same oscilloscope. In this way, the SAT measurements are immune to any phase misalignment between the two oscilloscope clocks.

The "electron-peak charge" $\left(Q_{e}\right)$ is defined by integrating a function that fits the electron-peak waveform. Digitizations between the start and the end points of the electron-peak waveform (i.e. the first points situated before and after the maximum whose amplitude is less than one standard deviation away from the baseline offset ${ }^{4}$ are fitted by a function, $\mathrm{f}(\mathrm{t})$, defined as the difference of two generalized logistic functions:

$$
f(t)=\frac{P_{0}}{\left[1+e^{-P_{1}\left(t-P_{2}\right)}\right]^{P_{3}}}-\frac{P_{0}}{\left[1+e^{-P_{4}\left(t-P_{5}\right)}\right]^{P_{6}}}
$$

The terms, $P_{0}, P_{1}, P_{2}, P_{3}, P_{4}, P_{5}$ and $P_{6}$ are free parameters in the fit of the electron-peak digitizations ( $\sim 40$ degrees of freedom). Eq. (1) is then numerically integrated and the resulting value is transformed to Coulombs, using the input impedance of $50 \mathrm{Ohm}$. The electron-peak charge-to-amplitude ratio, for all the instrumented PICOSEC-Micromegas pads, is measured to be around 0.029 $\mathrm{pC} / \mathrm{mV}$.

\section{Detector aligment}

We reported [16 that the uncertainty in measuring the arrival time of MIPs follows an "inverse-square-root" dependence on the number of photoelectrons, which initiate avalanches in the effective volume of the detector. In the case of a PICOSEC-Micromegas detector with segmented anode (Fig. 3), the timing information provided by each pad should depend on the way that the induced avalanches are shared between the effective area of neighboring pads. This sharing is determined by the MIP impact point on the detector plane relative to the centres of the pads. Thus, a precise knowledge of the detector position, relative to the global tracking coordinate system (hereafter called beam-frame), is necessary for evaluating the timing performance of the PICOSEC-Micromegas prototype.

Special care was taken in placing the PICOSEC-Micromegas detector in the experimental set-up with its radiator window perpendicular to the beam. The positions of the PICOSEC-Micromegas pad centres in the beam-frame are estimated off-line, by analysing the spatial distributions of the electron-peak charge $\left(Q_{e}\right)$.

A first estimation of a pad position is provided by the dependence of the average $Q_{e}$, induced by MIPs that impact the PICOSEC-Micromegas at $\left\{x_{B}, y_{B}\right\}$

\footnotetext{
${ }^{4}$ For those pulses with no clear separation between the electron-peak and the ion-tail, the end point has been alternatively defined as the time when the pulse derivative changes sign.
} 

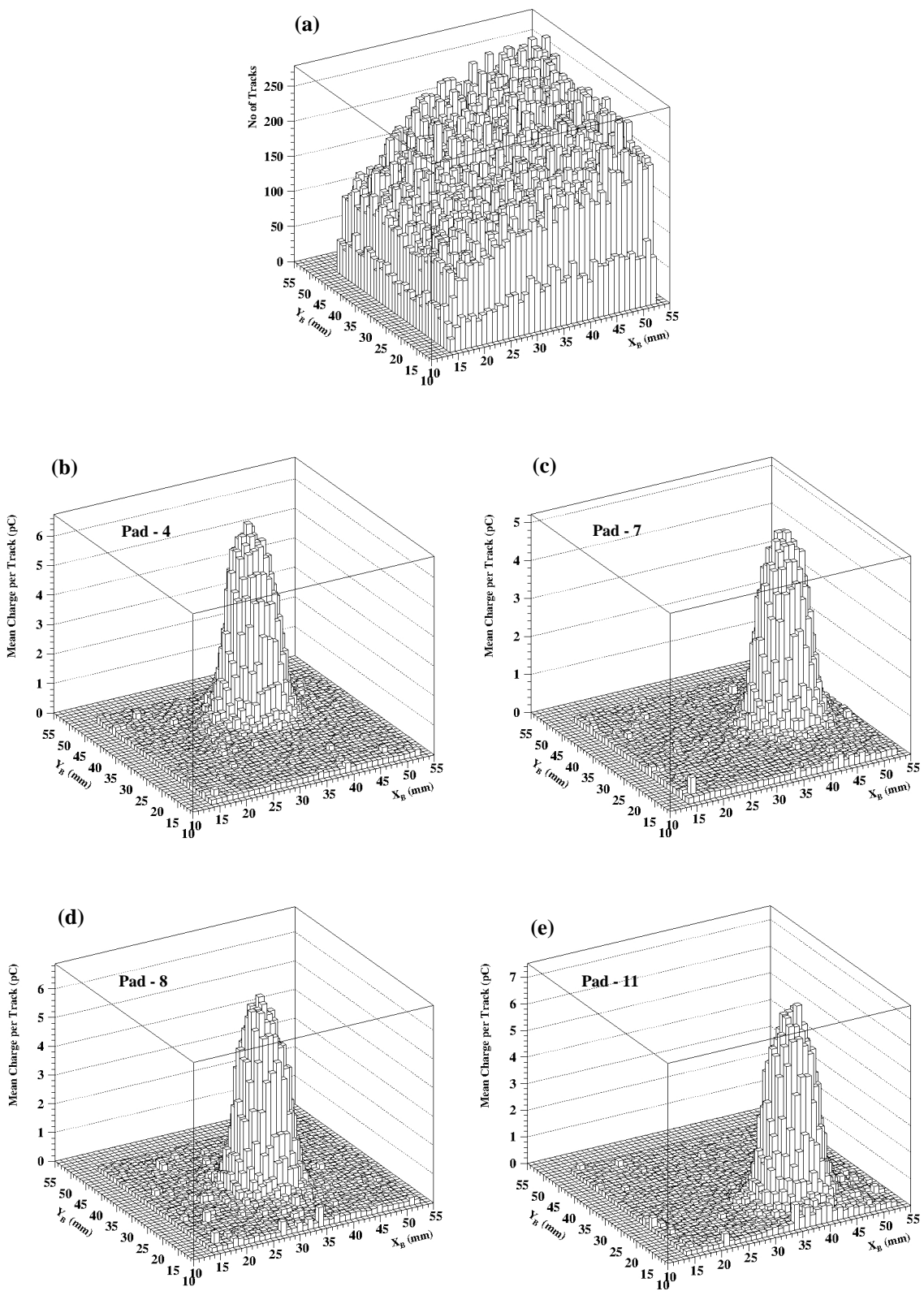

Figure 5: The 2D distribution in plot-a represents the beam profile that illuminates the area around the instrumented PICOSEC-Micromegas pads. The distributions below show the mean value of the electron-peak charge versus the $\left\{x_{B}, y_{B}\right\}$ coordinates where the MIPs traverse the detector. The distributions in plots b, c, d and e correspond to the response of pad No. 4, pad No. 7, pad No. 8 and pad No. 11, respectively. 
coordinates in the beam-frame. In principle, the centroid of each 2D-histogram shown in Fig. 5 provides an estimation of the respective pad center position, which is expressed in terms of the corresponding abscissa and bin contents as:

$$
x_{c}=\frac{\sum_{i=1, n}\left\langle Q_{e}\right\rangle_{i} x_{B}^{i}}{\sum_{i=1, n}\left\langle Q_{e}\right\rangle_{i}} \quad, \quad y_{c}=\frac{\sum_{i=1, n}\left\langle Q_{e}\right\rangle_{i} y_{B}^{i}}{\sum_{i=1, n}\left\langle Q_{e}\right\rangle_{i}}
$$

where $n$ is the number of bins, each bin corresponding to a $1 \times 1 \mathrm{~mm}^{2}$ area around a point with $\left\{x_{B}^{i}, y_{B}^{i}\right\}$ coordinates at the beam-frame and $\left\langle Q_{e}\right\rangle_{i}$ is the mean electron-peak charge for tracks with impact points in the $i^{\text {th }}$ bin.

As it is shown in Fig. 6, the $Q_{e}$ distributions related to the selected tracks are dominated by small pulses and noise; nevertheless, the $2 \mathrm{D}$ charge-weighted beam profiles (Fig. 5) clearly indicate the position of the pad centres. Furthermore, since the beam illuminates the whole active area of all PICOSECMicromegas pads the estimation by Eq. (2) is unbiased. However, because the two-dimensional binning of the events reduces the statistical accuracy of the estimation, Eq. (2) is only utilized to approximate the starting values, $\left\{x_{c}^{k}, y_{c}^{k}\right\}$ (hereafter, $\mathrm{k}=1,2,3,4$ stands for pad No. 4, 7, 8 and 11, respectively) for a more accurate estimation procedure.

We define the local-frame as the coordinate system with its origin at the centre of pad No. 8 and its axes, $X_{L}$ and $Y_{L}$, parallel to the principal, symmetry axes of the hexagonal pads (Fig. 3). In the local-frame, the pad centre coordinates $\left(\left\{x_{L}^{k}(d), y_{L}^{k}(d)\right\}, k=1,2,3,4\right)$ are completely defined in terms of the hexagonal pad dimensions and the width, $d$, of the gap between neighboring pads. Then, the pad centres coordinates $\left(\left\{x_{B}^{k}, y_{B}^{k}\right\}, k=1,2,3,4\right)$ in the beam-frame are expressed in terms of the local-frame variables as:

$$
\begin{aligned}
x_{B}^{k}\left(x_{o}, y_{o}, \vartheta, d\right) & =\left(x_{L}^{k}(d)-x_{o}\right) \cos (\vartheta)+\left(y_{L}^{k}(d)-y_{o}\right) \sin (\vartheta) \\
y_{B}^{k}\left(x_{o}, y_{o}, \vartheta, d\right) & =\left(y_{L}^{k}(d)-y_{o}\right) \cos (\vartheta)-\left(x_{L}^{k}(d)-x_{o}\right) \sin (\vartheta)
\end{aligned}
$$

where $\left\{x_{o}, y_{o}\right\}$ and $\vartheta$ are the coordinates of the beam-frame origin and the azimuth angle of the $X_{B}$ axis in the local-frame, respectively.

We use the coordinates of the centroids as "data points" (i.e. $x_{d a t a}^{k}=x_{c}^{k}$ and $y_{d a t a}^{k}=y_{c}^{k}$ for $\left.k=1,2,3,4\right)$ for an initial evaluation of the $x_{o}, y_{o}, \vartheta$ and $\mathrm{d}$ parameter values, by minimizing the following $\chi^{2}$ estimator:

$$
\chi^{2}=\sum_{k=1,4} \frac{\left(x_{d a t a}^{k}-x_{B}^{k}\left(x_{o}, y_{o}, \vartheta, d\right)\right)^{2}}{\left(\sigma_{x}^{k}\right)^{2}}+\sum_{k=1,4} \frac{\left(y_{d a t a}^{k}-y_{B}^{k}\left(x_{o}, y_{o}, \vartheta, d\right)\right)^{2}}{\left(\sigma_{y}^{k}\right)^{2}}
$$

\footnotetext{
${ }^{5}$ Also because, a) the hexagonal pad shape is $2 \mathrm{D}$ symmetric and b) the Cherenkov photons on the photocathode are symmetrically distributed around the MIP track.
} 

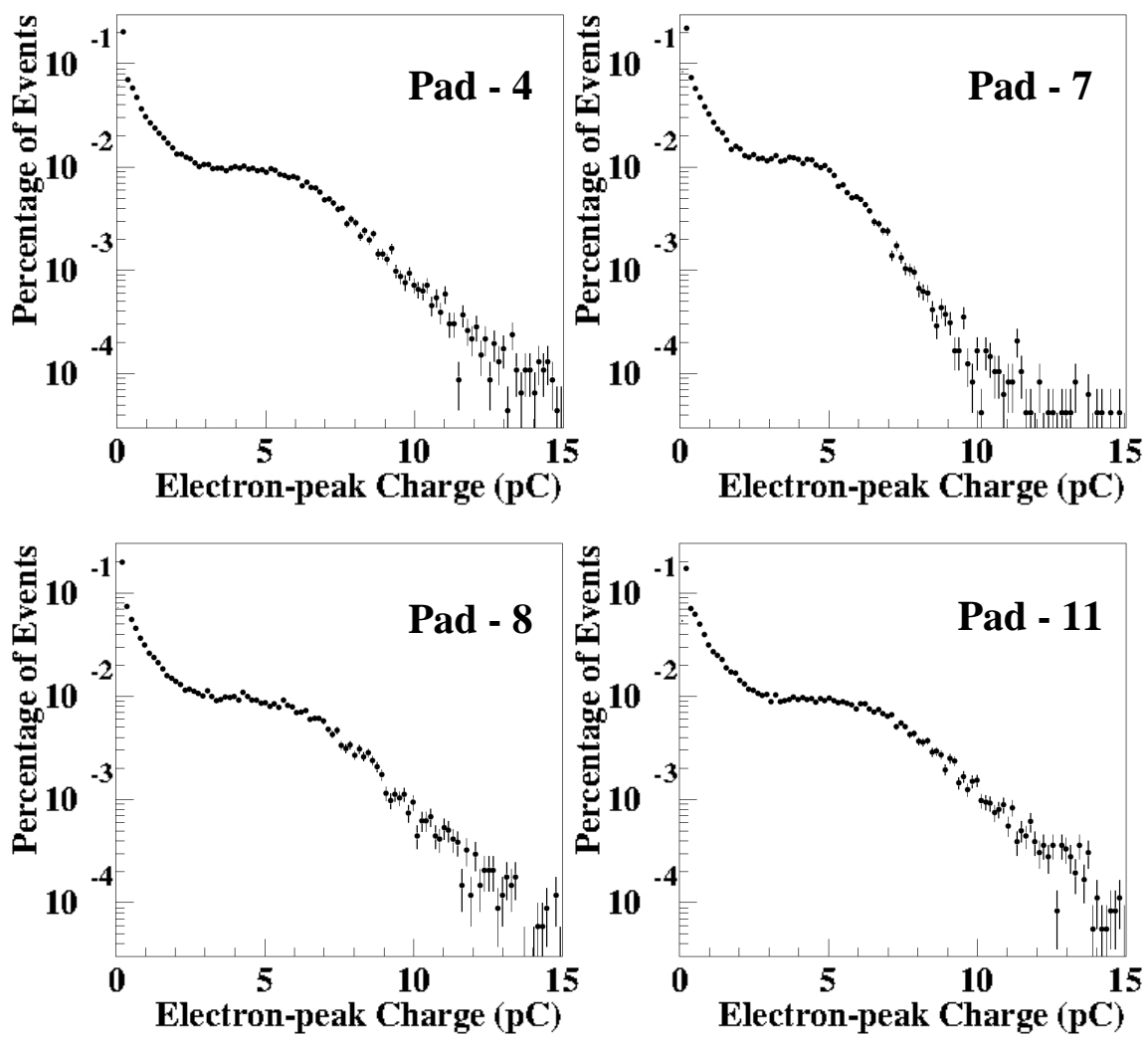

Figure 6: Electron-peak charge distributions of the instrumented pads, corresponding to tracks passing within $6 \mathrm{~mm}$ from the respective mean charge centroids (see text).

where $x_{B}^{k}\left(x_{o}, y_{o}, \vartheta, d\right)$ and $y_{B}^{k}\left(x_{o}, y_{o}, \vartheta, d\right)$ are given by Eq. 3 , whilst $\sigma_{x}^{k}$ and $\sigma_{y}^{k}$ are approximated by propagating the $\left\langle Q_{e}\right\rangle_{i}$ statistical errors in Eqs. $2 \sqrt{6}$. The estimated parameter values $\left(\hat{x}_{o}, \hat{y}_{o}, \hat{\vartheta}, \hat{d}\right)$ also define the pad centre positions in the beam-frame via Eq. (3).

The above estimations of the pad centre positions and of the azimuth angle, $\hat{\vartheta}$, are used as input values to a two-step, iterative algorithm. In the first step, each pad is treated independently; its centre coordinates are estimated

${ }^{6}$ That is $\sigma_{x}^{2}=\frac{\sum_{i=1, n} \delta_{i}^{2}\left(x_{c}-x_{B}^{i}\right)^{2}}{\sum_{i=1, n}\left\langle Q_{e}\right\rangle_{i}}$ and $\sigma_{y}^{2}=\frac{\sum_{i=1, n} \delta_{i}^{2}\left(y_{c}-y_{B}^{i}\right)^{2}}{\sum_{i=1, n}\left\langle Q_{e}\right\rangle_{i}}$, where $\delta_{i}$ denotes the statistical error in evaluating $\left\langle Q_{e}\right\rangle_{i}$ 

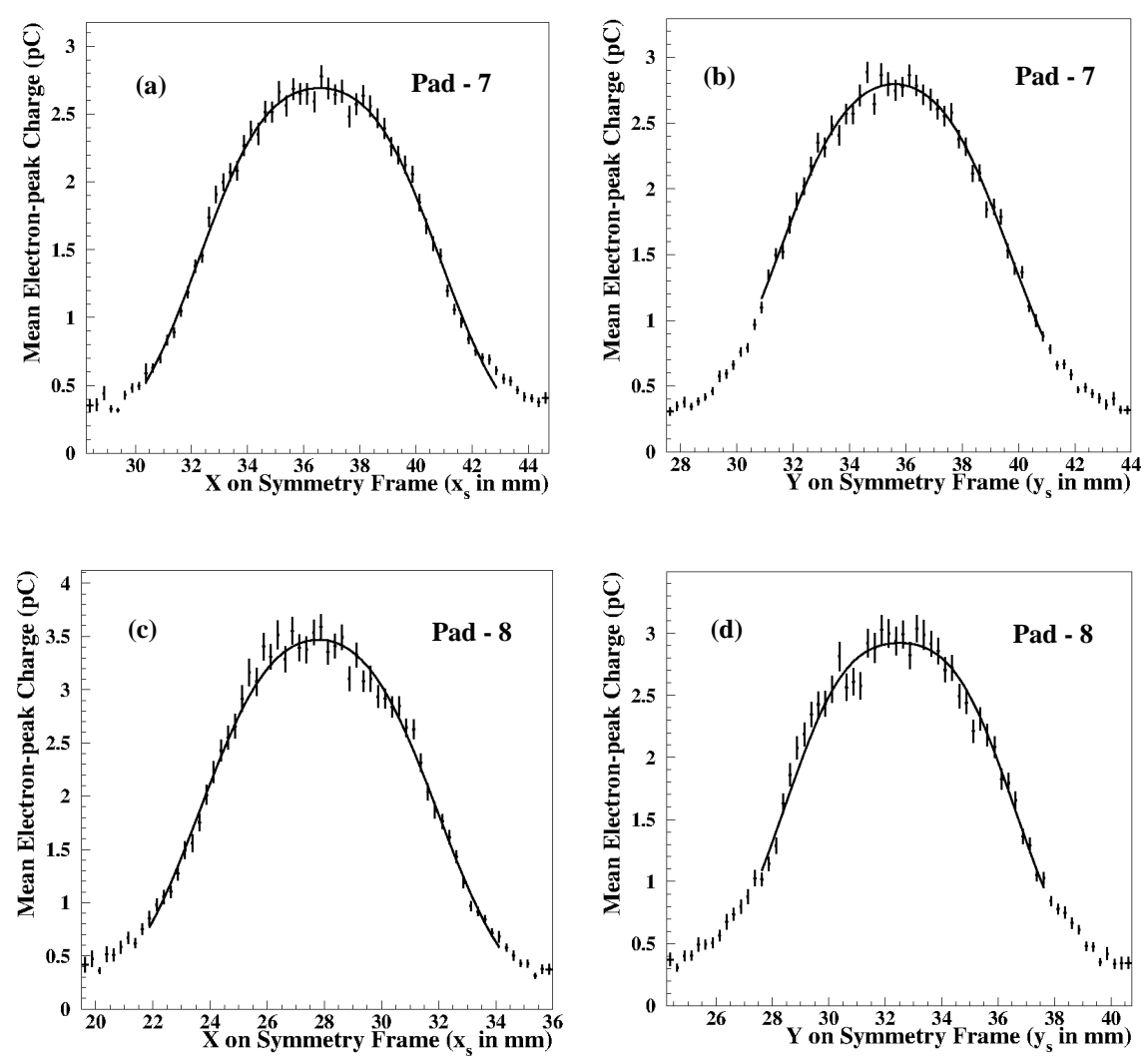

Figure 7: The mean value of the electron-peak charge, when the pad responds to MIPs entering the PICOSEC-Micromegas radiator at points with $x_{S}$ (plots a and c) or $y_{S}$ (plots b and d) coordinate, relative to the symmetry axes (see text). The upper and lower rows correspond to pads No. 7 and 8 respectively.

by fitting the charge-weighted, beam profiles projected to the pad principal, symmetry axes. The constraints arising from the anode segmentation geometry (Eq. (3p) are imposed in the second step, by minimizing the $\chi^{2}$ expression of Eq. (4) using the results of the first step as "data points".

First Step: Use the previously estimated values of the pad centre coordinates to select MIPs that pass through a disk of $6 \mathrm{~mm}$ radius around the pad centre. Use the previously estimated value of the angle $\vartheta\left(\vartheta=\hat{\vartheta}_{I}\right)$ to transform the beam coordinates to the symmetry axes, $X_{S}$ and $Y_{S}$ (Fig. 3):

$$
\begin{array}{r}
x_{S}^{k}=x_{B}^{k} \cos (-\vartheta)+x_{B}^{k} \sin (-\vartheta) \\
y_{S}^{k}=y_{B}^{k} \cos (-\vartheta)-x_{B}^{k} \sin (-\vartheta)
\end{array}
$$

Determine the $Q_{e}$ weighted beam profiles, projected along the $X_{S}$ and 
the $Y_{S}$ symmetry axes, i.e. the average $Q_{e}$ of the pad response to a MIP versus the respective $x_{S}$ or $y_{S}$ coordinate of the track impact point. Due to the symmetry of the anode segments with respect to the $X_{S}$ and $Y_{S}$ axes and the azimuthal symmetry of the Cherenkov disk, the above 1D profiles are symmetric around the respective centre coordinate (Fig. 7). We explore this symmetry by fitting Eq. (6) to the central part of the

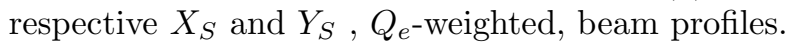

$$
\begin{array}{r}
\left\langle Q_{e}\right\rangle_{X_{S}}=N_{X}\left[e^{-\frac{\left(x_{S}-\mu_{x 1}\right)^{2}}{2 \varrho_{x}^{2}}}+e^{-\frac{\left(x_{S}-\mu_{x 2}\right)^{2}}{2 \varrho_{x}^{2}}}\right] \\
\left\langle Q_{e}\right\rangle_{Y_{S}}=N_{Y}\left[e^{-\frac{\left(y_{S}-\mu_{y 1}\right)^{2}}{2 \varrho_{y}^{2}}}+e^{-\frac{\left(y_{S}-\mu_{y 2}\right)^{2}}{2 \varrho_{y}^{2}}}\right]
\end{array}
$$

Accordingly, the pad centre coordinates at the $X_{S}$ and $Y_{S}$ axes are defined as, $0.5\left(\hat{\mu}_{x 1}+\hat{\mu}_{x 2}\right)$ and $0.5\left(\hat{\mu}_{y 1}+\hat{\mu}_{y 2}\right)$, respectively. The corresponding coordinates at the beam-frame are evaluated by the inverse transformation of Eq. (5). The procedure is applied for all instrumented pads of the PICOSEC-Micromegas prototype, resulting in independent estimations of the $x_{B}^{k}$ and $y_{B}^{k}(k=1,2,3,4)$ centre coordinates of all pads.

Second Step: Using the first step results (and the related estimation uncertainties) as "data points", the values of the $x_{o}, y_{o}, \vartheta$ and d parameters are estimated by minimizing the $\chi^{2}$ expression defined in Eq. (4). The pad positions in the beam-frame are then evaluated by inserting the above estimated values in Eq. (3).

The iterative process is considered complete when the pad positions found in the second step are the same, within the estimated statistical errors, with the results of the preceding first step. Otherwise, the values of the pad centres coordinates and of the angle $\vartheta$, found in this trial, are used as input values to the first step, for a new iteration.

We checked for possible systematic biases of the above method by: a) employing other symmetric functional forms instead of Eq. (6), b) selecting to fit different central regions of the $1 \mathrm{D} Q_{e}$-weighted beam profiles, and c) changing (by three sigmas) the starting values provided by the initial stage. The alignment algorithm converges each time after the second iteration, resulting consistently to almost the same estimation of the pad centre coordinates. The minimum $\chi^{2}$ per degree of freedom (p.d.f.) remains always around one, whilst the estimation errors, including the above systematic variations, are found to be less than $100 \mu \mathrm{m}$. However, the estimated value of the parameter $\mathrm{d}$ is found to be $430 \pm 70 \mu \mathrm{m}$, which is large compared to the design value $(\sim 200 \mu \mathrm{m})$. By setting the parameter d equal to $200 \mu \mathrm{m}$ the algorithm converges to coordinate 

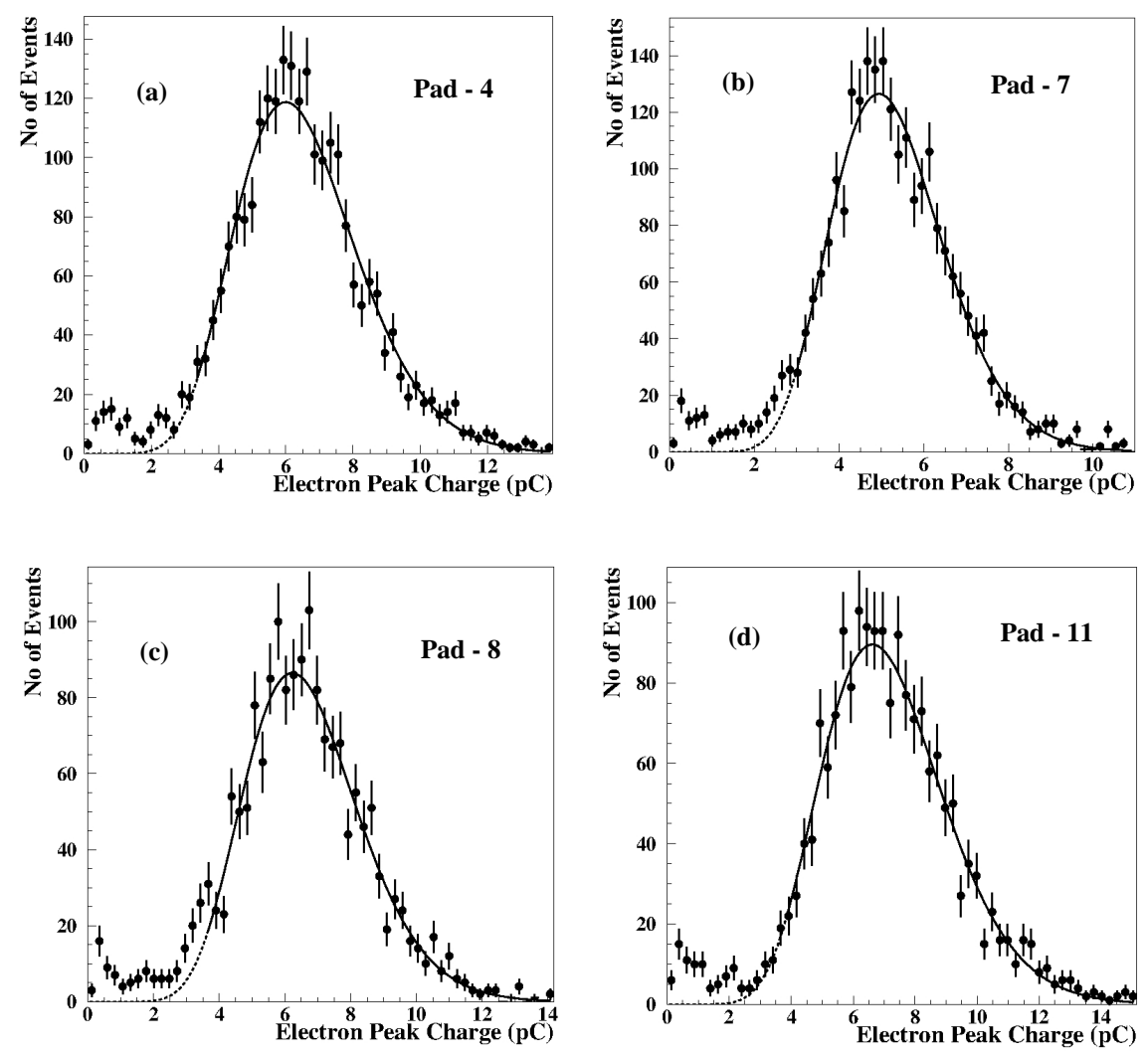

Figure 8: Electron-peak charge distributions of pads No. 4 (plot a), No. 7 (plot b), No. 8 (plot c) and No. 11 (plot d), when respond to MIPs passing closer than $2 \mathrm{~mm}$ from the respective pad centre. The solid lines represent fits with a Gamma Distribution function, with estimated mean values equal to $6.54( \pm 0.04) \mathrm{pC}, 5.29( \pm 0.03) \mathrm{pC}, 6.70( \pm 0.05) \mathrm{pC}$ and $7.20( \pm 0.05)$ $\mathrm{pC}$ for pads No. $4,7,8$ and 11 , respectively. 


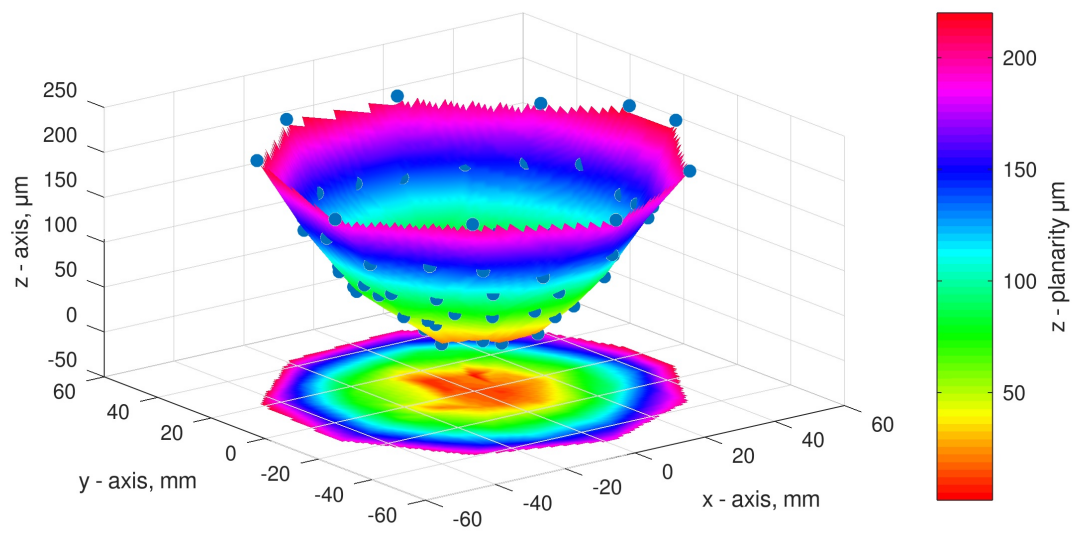

Figure 9: The radial deformation of the multi-pad PICOSEC-Micromegas anode has been measured with a ZEISS O-INSPECT 863 (length measurement error in $\mu \mathrm{m}: 2.2+\mathrm{L} / 150 \mathrm{~mm}$ ) at the CERN Metrology service. The shown measurements have been performed on the PCB installed on the aluminium flange.

values that differ by less than $100 \mu \mathrm{m}$ from the respective values when $\mathrm{d}$ was treated as a free parameter, whilst the minimum $\chi^{2}$ p.d.f. is getting more than three times larger.

Fig. 8 presents the $Q_{e}$ distributions of the instrumented PICOSEC-Micromegas pads, responding to MIPs with impact points within $2 \mathrm{~mm}$ from the respective pad centres, i.e. the vast majority of the Cherenkov induced signal is contained in a single pad. As evident in Fig. 6, the central pad No. 7 has a lower gain than the other pads (hereafter pads No. 4, 8 and 11 are referred as peripheral pads). We verified with laboratory tests that this difference is due to the spatial variation of the detector gain and it is independent of the external electronics. Microscopic inspection of the anode, revealed a non-flat, sagging surface with the lower tip close to the centre of pad No. 7, as it is shown in Fig. 9. As the micromesh is attached to the anode by the pillars, the depth of the amplification gap remains constant over the entire anode surface. However, as the cathode is planar, the preamplification (drift) gap is larger in the region above pad No. 7 than above the other pads. Therefore, despite the constant drift voltage, the drift field is lower above pad No. 7 as compared to the region above the peripheral pads, resulting in a gain variation as indicated in Fig. 8 Furthermore, as indicated in Fig. 9, the drift field would also vary across the surface of any peripheral pad, i.e. a preamplification avalanche that develops further away from pad No. 7 feels a higher drift field than those developing closer to the central pad. Such a gain asymmetry affects the alignment of the peripheral pads by introducing biases outwards from pad No. 7. These biases result in an overestimation of the gap width, d, between neighboring pads, as 
reported earlier. Although, the drift field variation across the anode surface causes minor effects on the detector alignment, it has significant consequences on the timing performance, discussed in the next Section.

\section{Timing characteristics and flatness corrections of individual pads}

PICOSEC-Micromegas is a standalone detector which performs precise timing measurements without requiring external information on the track of the incoming particle. In the case that the Cherenkov ring is shared among neighboring pads, a proper combination of the individual timing measurements (see Section 6), corrected for SAT shifts and weighted by their expected accuracy, provides precise timing of the incoming particles. However, the a priori knowledge of the individual measurements expected accuracy and time shifts is highly important for the combined timing. Since the timing accuracy and the SAT shift of a single pad depend explicitly on $Q_{e}$ [16, 6, 9, such dependences have to be quantified in a calibration phase prior to the detector operation.

Because the drift field of the prototype detector under study varies across the anode surface, the individual pads are expected to exhibit different timing behaviour. Specifically, due to the shape of the geometrical deformation discussed in Section 4 , the timing performance of the central pad is almost unperturbed but the variation of the drift-gap across the peripheral pads causes inhomogeneities to their timing precision and SAT. In this Section, we describe corrections that recover (almost) uniform timing characteristics across the entire anode surface.

Fig. 10 shows SAT distributions for the central pad (pad No. 7) and one of the peripheral pads (pad No. 11) when the tracks of incoming MIPs pass within $2 \mathrm{~mm}$ and between $2 \mathrm{~mm}$ and $4.33 \mathrm{~mm} 7^{7}$ of the respective pad centres. In the first case almost all the photoelectrons produced by a MIP contribute to the signal of the pad, while in the second case the pad signal is induced by a fraction of the photoelectrons. The time resolution of the central pad, quantified as the spread (RMS) of the corresponding SAT distribution, is $26.5 \pm 0.5 \mathrm{ps}$ and 34.0 $\pm 0.6 \mathrm{ps}$ in the first and in the second case, respectively. The peripheral pads perform with worse time resolution, i.e. $\sim 33.5 \mathrm{ps}$ and $\sim 49 \mathrm{ps}$ on average, for the above two categories of incoming MIP track 8 , respectively.

Moreover, the time resolution of the central pad is indeed an exclusive function of $Q_{e}$, irrespectively of the MIP impact point distance from the pad centre, as demonstrated in the left plot of Fig. 11. On the contrary, the time resolution of all the peripheral pads depends on both, $Q_{e}$ and the MIP track proximity to the pad centre. The right plot of Fig. 11 demonstrates this dependence for pad

\footnotetext{
${ }^{7}$ The radius of the inscribed circle into the hexagonal pad is $4.33 \mathrm{~mm}$.

${ }^{8}$ For MIPs passing $2 \mathrm{~mm}$ around the centres of pads No. 4, No. 8 and No. 11 the respective SAT spreads are $35.0 \pm 0.6 \mathrm{ps}, 33.5 \pm 0.6 \mathrm{ps}$ and $32.5 \pm 0.6 \mathrm{ps}$. For MIPs that pass between $2 \mathrm{~mm}$ and $4.33 \mathrm{~mm}$ from the pads centres the SAT spreads are $50.0 \pm 1.0 \mathrm{ps}, 48 . \pm 0.8 \mathrm{ps}$ and $48.5 \pm 1.0$ ps respectively.
} 

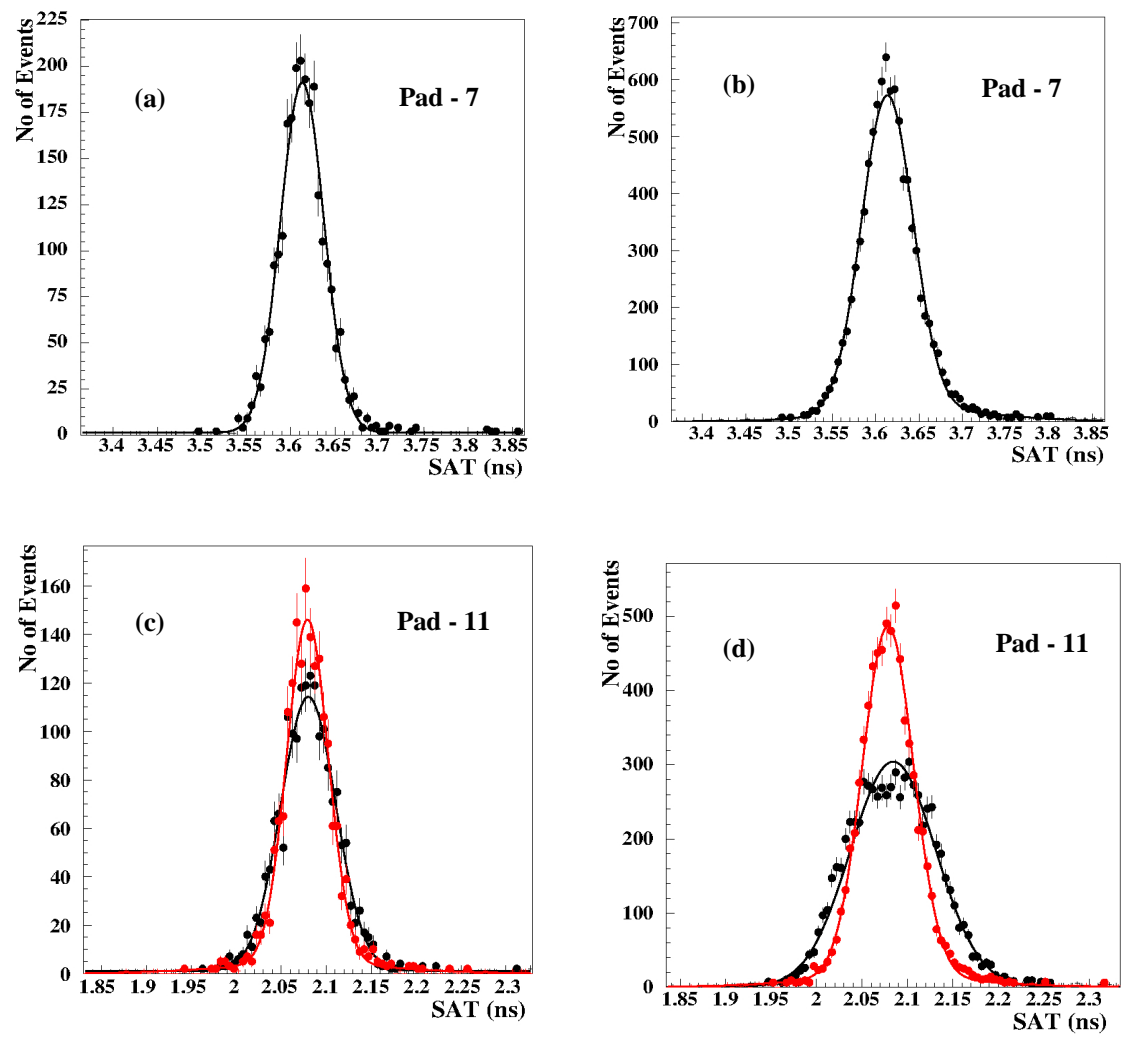

Figure 10: $\quad$ SAT distributions of pad No. 7 (plots a and b) and pad No. 11 (plots c and d) signals, corresponding to incoming MIPs passing within $2 \mathrm{~mm}$ (plots a and c) or between 2 $\mathrm{mm}$ and $4.33 \mathrm{~mm}$ (plots $\mathrm{b}$ and $\mathrm{d}$ ) from the respective pad centres. The black points represent raw SAT measurements while the red points show the same measurement distributions after applying the flatness corrections described later in Section 5 The solid lines represent fits to the data points with the sum of two Gaussian functions sharing the same mean value. 

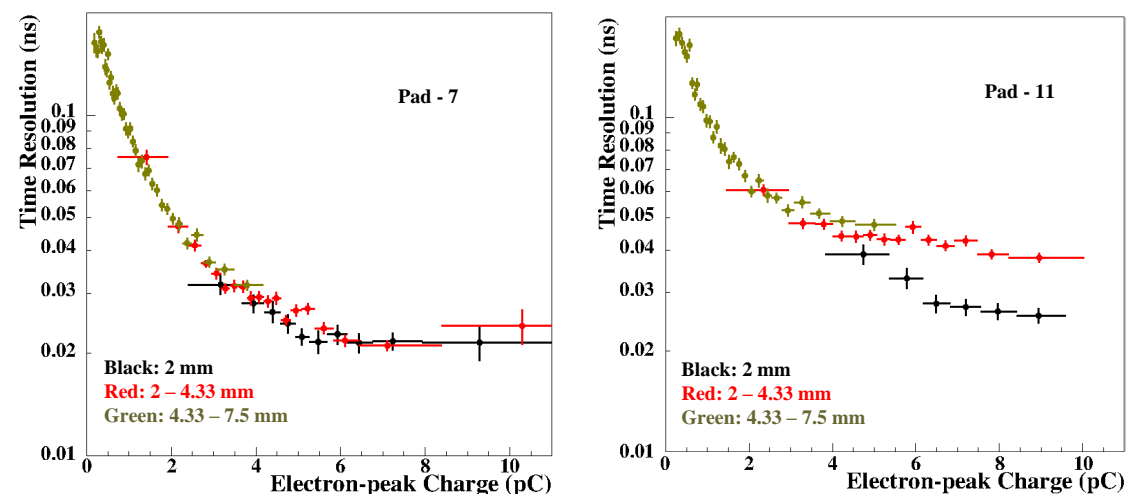

Figure 11: The time resolution of a single pad versus the respective $Q_{e}$ for MIPs passing within $2 \mathrm{~mm}$ (black), between $2 \mathrm{~mm}$ and $4.33 \mathrm{~mm}$ (red) and between $4.33 \mathrm{~mm}$ and $7.5 \mathrm{~mm}$ (green) from the pad centre, respectively. The left plot corresponds to the central pad No. 7 and the right plot to the peripheral pad No. 11.

No. 11, which is typical for any of the peripheral pads. MIPs passing closer than $2 \mathrm{~mm}$ from the centre of a peripheral pad are timed with better accuracy than MIPs passing further away, even though in both cases the induced signals have the same $Q_{e}$. In the following, we show that the above, undesirable, timing performance of the peripheral pads is mainly caused by the spatial inhomogeneity of the drift velocity, following the drift field variation across the anode surface.

Consider any axis passing through the centre of a pad (hereafter called test axis) and two points on this axis, $\mathrm{A}$ and $\mathrm{B}$, on either side and at equal distances from the pad centre, as shown in Fig. 12. In an ideal case, the timing information carried by the pad signals induced by MIPs passing from either of the above points should be identical. We found that the time resolution and the mean SAT values of the central pad respect the above, mirror symmetry. However, the mean SAT of the peripheral pads manifestly violates the symmetry with respect to the pad centres, as shown in Fig. 13 for the peripheral pad No. 8.

We define a reference axis, collinear to the line segment connecting the peripheral (in this case the pad No. 8) with the central pad centres and directing towards to the centre of the central pad, as illustrated in Fig. 12 In the rightmost, upper plot of Fig. 13, the chosen test-axis coincides with the reference axis for pad No. 8. The mean SAT values, corresponding to MIPs passing within $0.5 \mathrm{~mm}$ around certain points of the test-axis (hereafter called seeds), are plotted versus the coordinate of the respective seed along the test-axis, i.e. the signed distance from the pad centre. The peripheral pad signals induced by MIPs that pass around seeds with negative coordinates arrive faster than signals induced by tracks passing by their mirror symmetric (positive) seeds.

We examined the above mean SAT asymmetry along several test-axes, keep- 


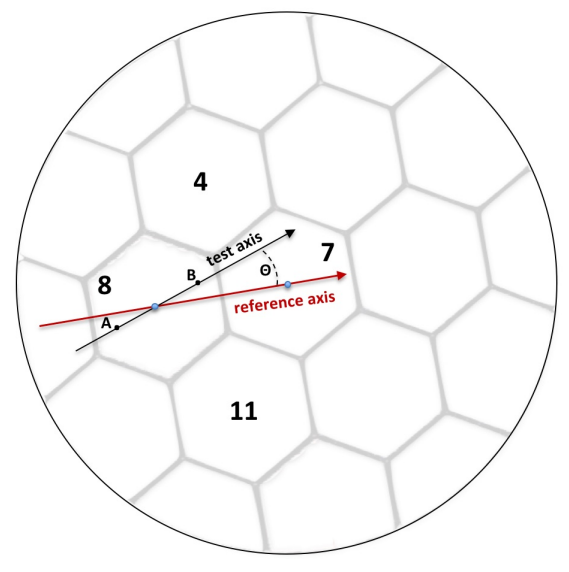

Figure 12: Illustration of the test and reference axes used for investigating the symmetry properties (see text) of the mean SAT and the time resolution of a single pad.

ing always the axis origin at the centre of the peripheral pad and turning the axis orientation by an angle, $\Theta$, with respect to the reference axis (see Fig. 12). The plots in Fig. 13 present mean SAT asymmetries along six test-axes, where positive (negative) $\Theta$ values indicate anti-clockwise (clockwise) turns. For all peripheral pads, the asymmetry is larger at small absolute values of the angle $\Theta$ while at $\Theta=90^{\circ}$ is significantly reduced. This mean SAT asymmetry reflects the spatial variation of the drift velocity, which in turn maps the variation of the drift field due to the geometrical deformation of the anode and the nonuniformity of the drift gap thickness. An almost spherical anode surface (as discussed in Section 40, with its lower tip close to the centre of the central pad, is consistent with the observed SAT asymmetries, i.e. with the observation that the SAT asymmetry is maximal along the direction defined by the peripheral and central pad centres whilst is minimal along the perpendicular direction.

However, by examining the RMS values of the SAT distributions (i.e. the time resolution) along the same test-axes we found that, unlike the mean SAT asymmetry, the time resolution of the peripheral pads are, in a good approximation, symmetric around the pad centres for all angles $\Theta$, as shown in Fig. 14.

The observed timing characteristics, shown in Figs. 13 and 14 , indicate that the spatial variation of the drift gap thicknes:9, although it affects the uniformity of the detector drift field which causes variations on the drift velocity, it does not have a major influence on the symmetry of the time resolution around the pad centre. However, the imposed spatial variation on the drift velocity causes local systematic shifts on the SAT.

\footnotetext{
${ }^{9}$ Notice that a change in the drift gap thickness besides of changing the drift field also affects the maximum available length for the preamplification avalanches evolution.
} 

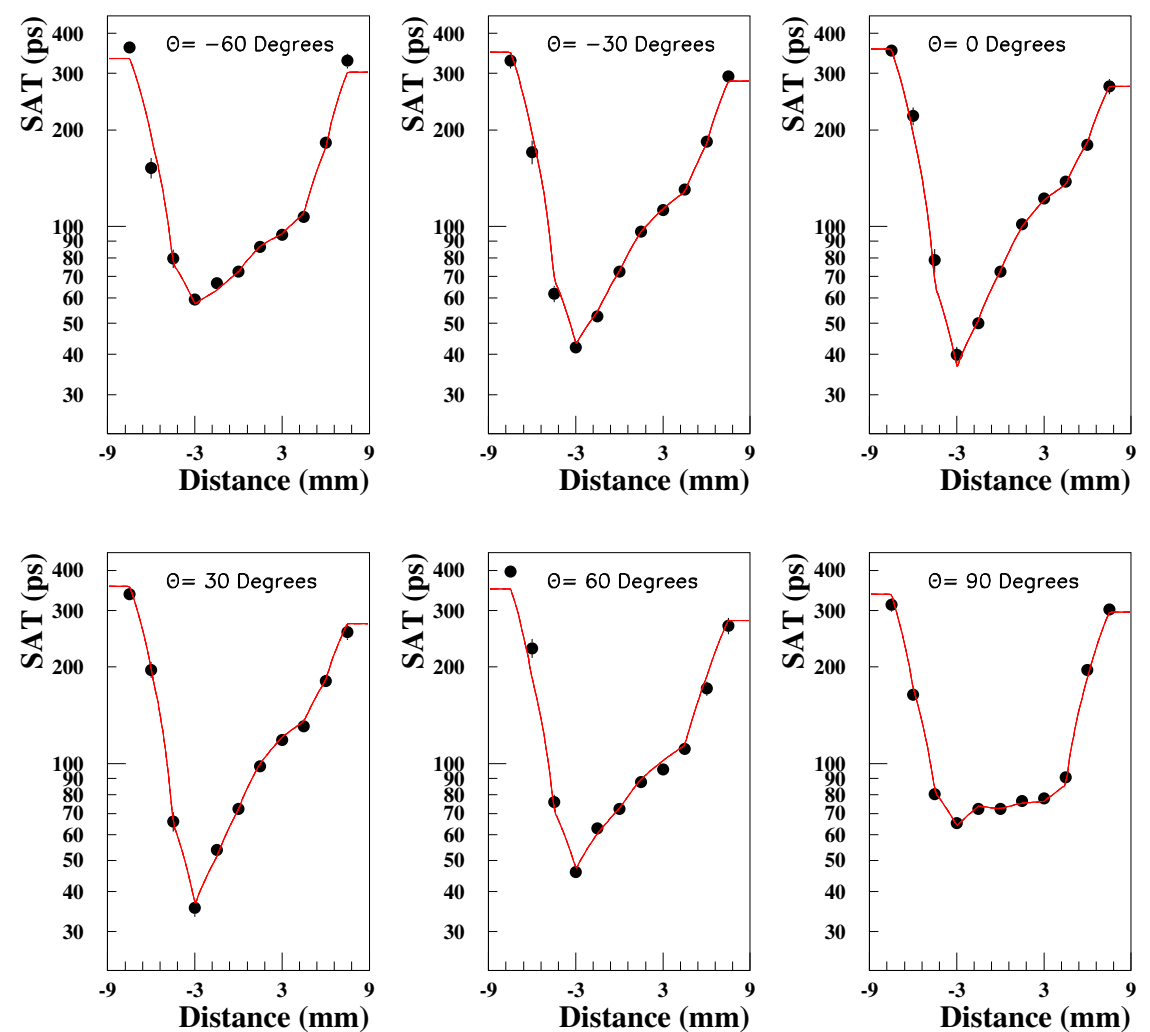

Figure 13: The mean signal arrival time (SAT) induced by MIPs to pad No. 8 versus the coordinates of the respective seeds on the test axis. Each point in the graphs corresponds to tracks passing within $0.5 \mathrm{~mm}$ around a seed, as it is described in the text. Every plot is marked by a value of the angle $\Theta$, which is the angle between the respective test-axis and the reference axis for pad No. 8. The solid lines represent empirical parametrizations. (For a better illustration, an offset of $2.019 \mathrm{~ns}$ has been subtracted from all the raw SAT measurements used in the plots) 

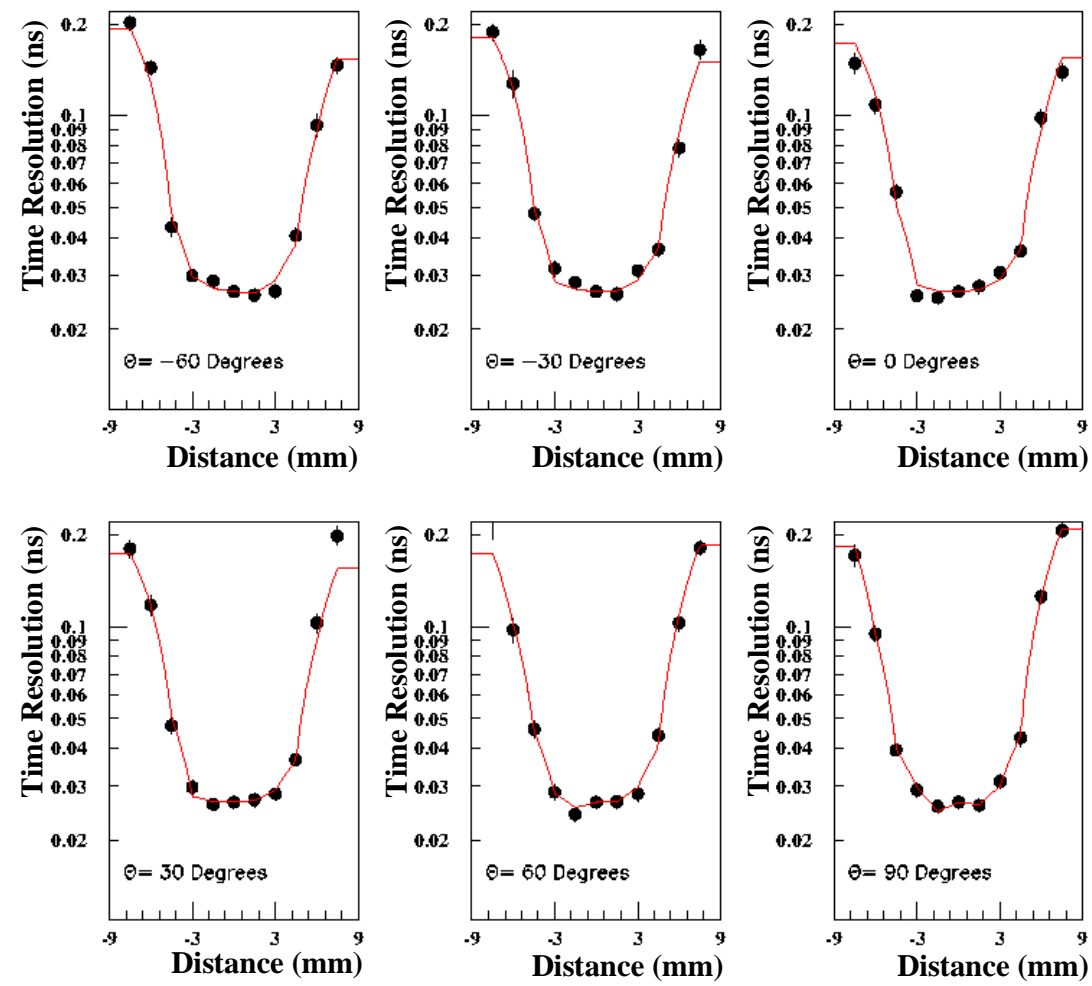

Figure 14: The spread (RMS) of SAT distributions induced by MIPs to pad No. 4 versus the coordinates of the respective seeds on the test axis. Each point in the graphs corresponds to tracks passing within $0.5 \mathrm{~mm}$ around a seed, as it is described in the text. Every plot is marked by a value of the angle $\Theta$, which is the angle between the respective test-axis and the reference axis for pad No. 4. The solid lines represent empirical parametrizations. 

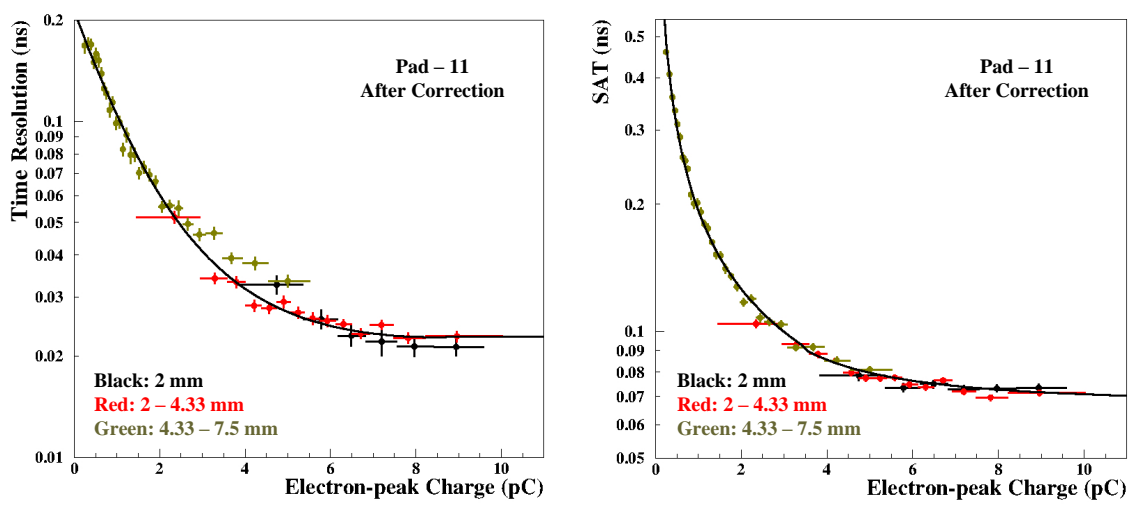

Figure 15: (left) The time resolution versus $Q_{e}$, after applying the flatness correction to signals of pad No. 11. (right) The mean SAT versus $Q_{e}$, after applying the flatness correction to signals of pad No. 11. For better illustration, an offset of 2 ns has been subtracted from the SAT values. Both plots follow the same colour code as in Fig. 11 to denote the regions of proximity of the respective MIP impact points to the pad centre. The solid curves represent fits to the data points from all proximity regions.

We developed corrections (hereafter called flatness corrections) in order to remove the timing biases caused by the geometrical deformation. For each peripheral pad (k), we parametrized the mean SAT values, e.g. those presented in Fig. 13. as a function of the cylindrical coordinates of the respective seed in the padframe $e^{10} S^{k}(r, \Theta)$. A correction factor, $\Delta^{k}(r, \Theta)=S^{k}(r, \Theta)-S^{k}\left(r, \Theta=90^{\circ}\right)$, is defined based on the observation that the SAT is almost mirror symmetric along the axis with $\Theta=90^{\circ}$. Then, the flatness correction is applied to the arrival times of all signals of the $k^{\text {th }} \mathrm{pad}$, as:

$$
T_{f-c o r r .}^{k}=T_{S A T}^{k}(r, \Theta)-\Delta^{k}(r, \Theta)
$$

where $T_{S A T}^{k}(r, \Theta)$ is the raw arrival time of the $k^{\text {th }}$ pad signal, which is induced by a MIP that impacts the PICOSEC-Micromegas plane at $(r, \Theta)$, and $T_{f-c o r r}^{k}$. is the SAT value corrected from biases due to geometrical distortions. It should be noticed that Eq. (7) is an empirical correction, which is based on several approximations, e.g. when we evaluate the parametrization $S^{k}(r, \Theta)$ the coordinates $(r, \Theta)$ refer to the seed points but when we use the flatness correction factors, $\Delta^{k}(r, \Theta)$, the coordinates refer to the specific impact point of the incoming MIP. Nevertheless, when the flatness correction is applied to the data, the timing properties of the peripheral pads approach those of the central pad.

\footnotetext{
${ }^{10}$ The pad-frame is a coordinate system with its origin at the centre of the pad and the reference axis defines the $\mathrm{X}$ axis of the frame. Notice that the azimuth angle in this frame coincides with the angle $\Theta$.
} 

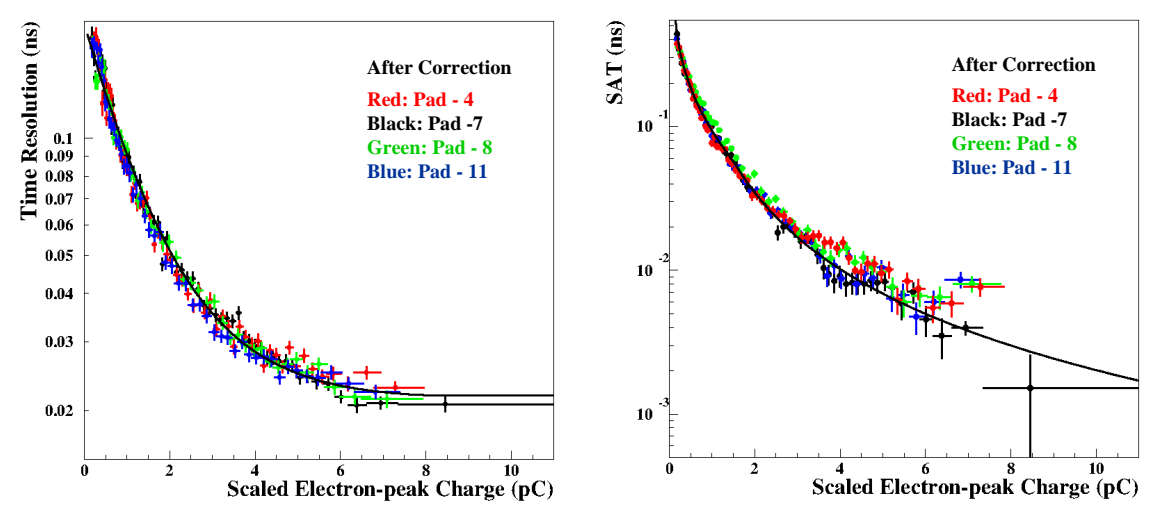

Figure 16: The time resolution (left) and the mean SAT (right), after applying the flatness correction to the peripheral pad signals, as a function of the scaled electron-peak charge (see text). External time delays have been subtracted from the SAT measurements of each pad. The black points correspond to the central pad while the other, coloured points correspond to the peripheral pads. The solid curves represent fits of the central pad data.

The effect of the flatness correction is demonstrated in Fig. 10. The red points represent, restored by Eq. (7), SAT distributions of the peripheral pad No. 11. After the flatness correction the time resolution for MIPs passing through the inner and outer region around the pad centre is $27.0 \pm 0.7 \mathrm{ps}$ and $35.2 \pm 0.5 \mathrm{ps}$, respectively, in a very good agreement with the central pad performance. Furthermore, as Fig. 15 demonstrates, after applying the flatness correction the time resolution of the peripheral pads versus $Q_{e}$ depends exclusively on the electron-peak charge, irrespectively of the proximity of the MIP track to the pad centre. Similarly, after applying the corrections, the mean SAT also depends exclusively on $Q_{e}$, independently on the MIP impact position.

In addition, by taking into account the gain difference between pads, i.e. by scaling down ${ }^{11}$ the electron-peak charge of the peripheral pads, the time resolution and the mean SAT dependences on the (scaled) $Q_{e}$ becomes almost the same for all pads, as it is illustrated in Fig. 16 .

Naturally, a multi-pad PICOSEC-Micromegas detector with a perfectly flat anode will exhibit the above timing characteristics without the need of any flatness correction. Nevertheless, due to the physical processes that produce the signal [9], the PICOSEC-Micromegas SAT depends on the electron-peak charge. Such a dependence should be evaluated a priori (calibration) and must be taken into account for precise timing measurements. In Fig. 16, the solid lines represent fits to the experimental points, providing also the functional

\footnotetext{
${ }^{11}$ The mean values of the electron-peak charge distributions, shown in Fig. 8 are used to determine the respective scaling factors.
} 

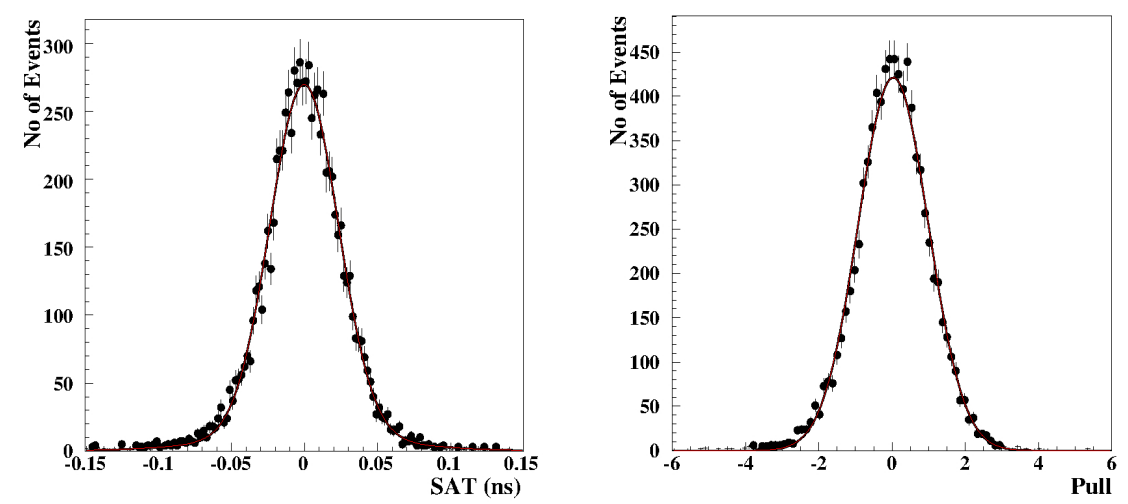

Figure 17: (left) Distribution of fully corrected arrival time measurements of all pad signals induced by MIPs passing within $2 \mathrm{~mm}$ from the respective pad centre. The solid line represent a double Gaussian fit to the data points with an RMS of $25.8 \pm 0.6$ ps. (right) Distribution of the fully corrected SAT measurements normalized to their expected error (pull). The solid line represents a Gaussian fit with estimated mean and $\sigma$ values consistent to 0 and 1 respectively.

description of the SAT (and of the time resolution) dependence on $Q_{e}$, i.e. $\tau\left(Q_{e}\right)$ (and $\sigma\left(Q_{e}\right)$ ). The left plot in Fig. 17 presents the distribution of fully corrected SAT values, i.e $T_{f-c o r r}^{k}-\tau\left(Q_{e}\right)$, for all the available single pad measurements, when the MIPs are passing within $2 \mathrm{~mm}$ from the respective pad centre. The global time resolution of the prototype multi-pad-PICOSEC-Micromegas, i.e. includind all pads, provided by the spread (RMS) of this distribution, is $25.8 \pm 0.6$ ps. The right plot illustrates the pull (i.e. $\frac{T_{f-c o r r}^{k}-\tau\left(Q_{e}\right)}{\sigma\left(Q_{e}\right)}$ ) distribution which is consistent with a standard normal distribution, signifying also the consistency in the evaluation of the expected resolution of a pad as a function of $Q_{e}$.

\section{Combining the timing information of several pads}

We consider cases when the Cherenkov ring of a MIP produces signals on more than one $(M>1)$ pad. After applying the flatness correction we assume that the pad signals share the same timing properties, as it is shown in Fig 16 . The dependence of the mean SAT and of the time resolution on the respective $Q_{e}$ have been calibrated and quantified by the functions $\tau\left(Q_{e}\right)$ and $\sigma\left(Q_{e}\right)$, respectively. Henceforth, we assume that each of the pad signals carries an independent information on the MIP arrival time. Then, we combine the singlepad measurements to form the following $\chi^{2}$ estimator:

$$
\chi^{2}=\sum_{m=1, M} \frac{\left(T_{\text {comb. }}-\left[T_{f-\text { corr. }}^{m}-\tau\left(Q_{e}^{m}\right)\right]\right)^{2}}{\sigma^{2}\left(Q_{e}^{m}\right)}
$$



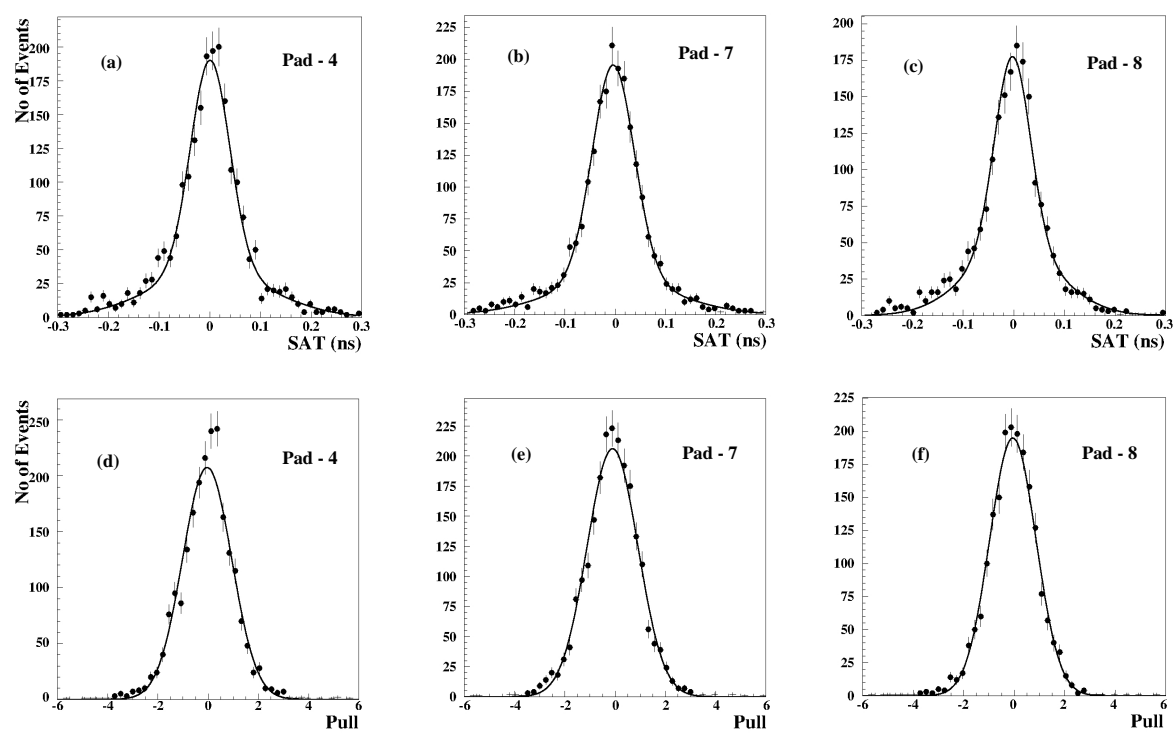

Figure 18: Distributions of fully corrected arrival time measurements of signals (SAT) induced on the pads No. 4 (plot a), No. 7 (plot b) and No. 8 (plot c) by MIPs passing within $2 \mathrm{~mm}$ of their common corner (see text). The solid lines represent fits of the data points with the sum of two Gaussian functions. The mean value of the distributions is consistent with zero, as it is expected after correcting for shifts by subtracting the calibration function $\tau\left(Q_{e}^{m}\right)$. The corresponding RMS values are $71.3 \pm 2.5,66.5 .0 \pm 2.5$ and $68.0 \pm 2.5$ ps for pads No. 4 , 7 and 8 , respectively. The plots $\mathrm{d}$, e and f show the corresponding pull distributions of the fully corrected single-pad SAT measurements normalized to the expected measurement errors. The solid lines represent Gaussian fits, which are consistent with standard normal distribution functions. 

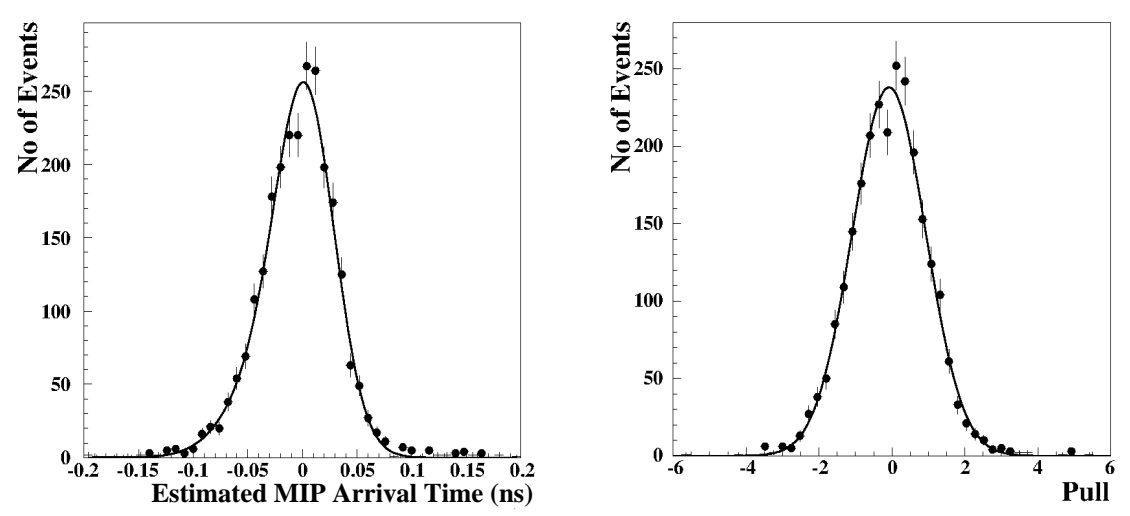

Figure 19: (left) Distribution of the arrival time of MIPs, passing within $2 \mathrm{~mm}$ of a common pad corner (pads No. 4, 7 and 8), estimated by Eq. (9) combining the individual single-pad measurements and their expected errors. The solid line represents a fit to the data points by a sum of two Gaussian functions corresponding to an RMS of $32.2 \pm 0.5$ ps. (right) Pull distribution of estimated arrival times by Eq. 9p. The solid line represents a Gaussian fit to the data points, consistent with mean and $\sigma$ values equal to 0 and 1 respectively.

where, $m=1,2, \ldots, M$ is an index identifying the pads sharing the Cherenkov ring. The minimization of the above $\chi^{2}$ provides an estimation of the MIP arrival time as:

$$
\hat{T}_{\text {comb. }}=\frac{\sum_{m=1, M} \frac{\left(T_{f-\text { corr. }}^{m}-\tau\left(Q_{e}^{m}\right)\right)^{2}}{\sigma^{2}\left(Q_{e}^{m}\right)}}{\sum_{m=1, M} \frac{1}{\sigma^{2}\left(Q_{e}^{m}\right)}}
$$

whilst the estimation uncertainty is evaluated by error propagation.

For a demonstration, Eq. (8) is applied to estimate the arrival time of MIPs passing within $2 \mathrm{~mm}$ from the common corner of pads No. 4, 7 and 8 . In this case, the photoelectrons are shared almost equally between the three anode segments and the induced signals are of similar amplitude. The top row of Fig. 18 shows the SAT distributions of each of the pads separately, corrected for shifts due to the $Q_{e}$ dependence (i.e. $T_{f-c o r r}^{m}-\tau\left(Q_{e}^{m}\right)$ ). In this case, the spread (RMS) of a single pad distribution, i.e. the resolution in timing the MIP arrival using a single-pad measurement, is about 68 ps. Moreover, the observed single-pad time resolution is in a good agreement with the expected resolution, which is expressed by the calibration function $\sigma\left(Q_{e}^{m}\right)$, as it is demonstrated by the pull (i.e. $\left.\frac{\left(T_{f-c o r r .}^{m}-\tau\left(Q_{e}^{m}\right)\right)}{\sigma\left(Q_{e}^{m}\right)}\right)$ distributions shown at the bottom row of Fig. 18 . 
Then, the MIP arrival time is also estimated by combining the individual single-pad measurements and their expected errors using Eq. (9). The distribution of the above estimations is shown in Fig. 19 (left plot) with a mean value that is consistent with zero, demonstrating the no-bias of the timing procedure. The accuracy in estimating the MIP arrival time is expressed by the RMS of the distribution, which is $32.2 \pm 0.5$ ps. Moreover, by evaluating the statistical error of each estimation by Eq. (99), we form the pull of the respective arrival time estimation from its true value. The emerging pull distribution, shown in the right plot of Fig. 19, follows a standard normal shape, signifying that the estimation error is consistently evaluated on an event by event basis.

For completeness, we monitor the accuracy in estimating the MIP arrival time as its track impacts the detector along the line connecting the centres of pad No. 11 and pad No. 4. The left plot in Fig. 20 shows the sampling-points (blue marks) used to select MIPs passing around them within $1 \mathrm{~mm}$ distance (e.g. the red circle represents such a region). We label these sets of tracks by the distance L of the respective sampling-point to the centre of pad No. 11. In the right plot of Fig. 20, the dots connected with red line segments represent the resolution in estimating the MIP arrival time by combining information from the active pads using Eq. (9) versus the distance L. The points connected with blue line segments illustrate the best single-pad timing performance (resolution) among the active anode segments. Depending on how the photoelectrons are shared between the neighboring segments, the time resolution is by $5-8 \mathrm{ps}(\sim 4$ sigma) worse than in the case that all the Cherenkov photons are measured by a single pad. This difference, however small, is possibly due to the limitations of the empirical flatness corrections, detailed in Section 5, to fully correct and equalize the timing performance of the prototype PICOSEC-Micromegas pads. Nevertheless, the worsening of the time resolution maybe also due to other effects such as: a distortion of the amplification field in the vicinity of the gaps between pads resulting to inefficiencies and extra timing errors or effects (e.g. cross talk between adjacent anode segments) which cause correlation between neighboring pad measurements. This type of possible effects will be investigated in the near future with a new, improved flatness, multi-pad PICOSEC-Micromegas prototype currently under construction.

\section{Concluding Remarks}

We developed a multi-pad PICOSEC-Micromegas prototype consisting of 19 hexagonal pads and we evaluated its performance with data collected in a muon test beam over 4 instrumented pads. In the present paper, we studied in detail the effect of the drift gap thickness non-uniformity on the time resolution and devised empirical flatness corrections to obtain a uniform timing response over the whole detector coverage. We measured a time resolution of $25.8 \pm 0.6$ ps when the muons impact the detector close to a pad center. However, the timing error rises up to $32.2 \pm 0.5$ ps when the track approaches the pad corners and the Cherenkov ring is shared among three pads. This increase is possibly 

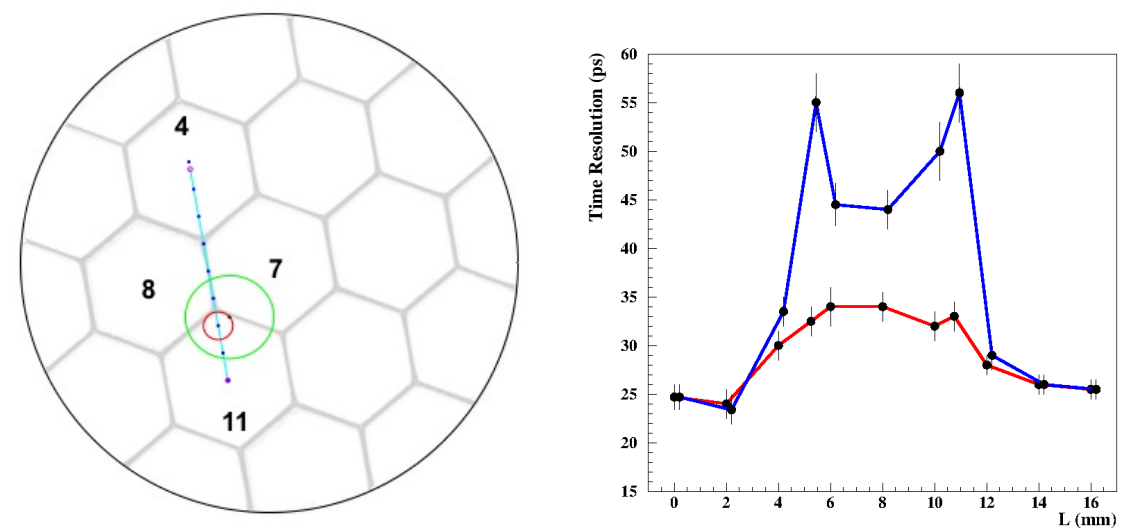

Figure 20: The resolution of the multi-pad PICOSEC-Micromegas prototype in timing the arrival of MIPs that impact the detector plane along the line connecting the centres of pad No. 11 and pad No. 4. In the left plot, the red circle, $1 \mathrm{~mm}$ in radius, displays the area used to select MIPs associated with the sampling-point shown at its centre. The green circle, $3 \mathrm{~mm}$ in radius, illustrates the Cherenkov disc of a track passing through a point on the periphery of the red circle. In the right plot, the dots connected by red line segments represent the resolution in timing the arrival of MIPs passing around a sampling-point at distance L from the centre of pad No. 11. The dots connected with blue line segments denote the best singlepad timing performance among the active anode pads. The points at $\mathrm{L}=5.25 \mathrm{~mm}$ and $\mathrm{L}=$ $10.75 \mathrm{~mm}$ display the respective time resolution for tracks passing around the common corners of three pads.

due to remaining systematics, i.e. to the limitation of the applied corrections to fully mitigate the systematic effects caused by the drift field non-uniformity. Nevertheless, there may be other effects contributing to the worsening of the time resolution when the Cherenkov photons initiate avalanches close to the pad edges (e.g. field distortions, cross-talk, etc). Such effects, introducing inefficiencies or/and correlations between adjacent pads, will be investigated in the near future with a new improved detector.

The Micromegas PCB of the current prototype suffers from initial deformations (see Fig. 9), most likely caused by the production process and mesh stretching, which can be additionally pronounced by tightening the PCB to the detector housing. In a new prototype, currently under construction, special care is taken to ensure precise flattening and polishing (e.g. by using more rigid and thicker PCB that combines a ceramic core for the rigidity and thin FR4 outer layers). Such an improved detector will have a drift thickness uniformity of better than $10 \mu \mathrm{m}$ across the area in front of the Cherenkov radiator and flatness corrections will not be needed. 


\section{Acknowledgments}

We acknowledge the support of the RD51 collaboration in the framework of RD51 common projects, the support of Photek Ltd. for lending us the MCPPMT during the beam tests and the financial support of the Cross-Disciplinary Program on Instrumentation and Detection of CEA, the French Alternative Energies and Atomic Energy Commission. M. Gallinaro acknowledges the support from the Fundação para a Ciência e a Tecnologia (FCT), Portugal. S. White acknowledges partial support through the US CMS program under DOE contract No. DE-AC02-07CH11359.

\section{References}

[1] M. C. S. Williams, Particle identification using time of flight, Journal of Physics G: Nuclear and Particle Physics 39 (12) (2012) 123001. doi:10. 1088/0954-3899/39/12/123001.

[2] E. Curras, et al., Radiation hardness and precision timing study of silicon detectors for the CMS high granularity calorimeter (HGC), Nucl. Instrum. Meth. A 845 (2017) 60-63. doi:10.1016/j.nima.2016.05.008.

[3] J. Caravaca, et al., Experiment to demonstrate separation of cherenkov and scintillation signals, Phys. Rev. C 95 (2017) 055801. doi:10.1103/ PhysRevC.95.055801

[4] R. Lipton, J. Theiman, Fast timing with induced current detectors, Nucl. Instrum. Meth. A 945 (2019) 162423. doi:https://doi.org/10.1016/j. nima.2019.162423.

[5] M. Centis Vignali, et al., Deep diffused apds for charged particle timing applications: Performance after neutron irradiation, Nucl. Instrum. Meth. A 949 (2020) 162930. doi:https://doi.org/10.1016/j.nima. 2019.162930.

[6] J. Bortfeldt, et al. for the RD-51 PICOSEC Collaboration, PICOSEC: Charged particle timing at sub-25 picosecond precision with a Micromegas based detector, Nucl. Instrum. Meth. A 903 (2018) 317-325.

[7] L. Sohl, et al. for the RD-51 PICOSEC Collaboration, Single photoelectron time resolution studies of the picosec-micromegas detector, Vol. 15, 15th Topical Seminar on Innovative Particle and Radiation Detectors (IPRD19), J. Instrum., 2020, p. C04053. doi:10.1088/1748-0221/15/04/C04053.

[8] Y. Giomataris, P. Rebourgeard, J. Robert, G. Charpak, MICROMEGAS: A high granularity position sensitive gaseous detector for high particle flux environments, Nucl. Instrum. Meth. A 376 (1996) 29-35. 
[9] J. Bortfeldt, et al. for the RD-51 PICOSEC Collaboration, Modeling the timing characteristics of the picosec micromegas detector, arXiv:1901.10779v2 (2020).

[10] Y. Giomataris, et al., Micromegas in a bulk, Nucl. Instrum. Meth. A 560 (2006) 405-408.

[11] PMT240/Q/BI/NG, http://www.photek.com/pdf/user-guides/ Photek--Photomultiplier-and-Photodiode--UserGuide.pdf (2016).

[12] J. Bortfeldt, et al. for the RD-51 PICOSEC Collaboration, Timing performance of a micro-channel-plate photomultiplier tube, Nucl. Instrum. Meth. A 960 (2020) 163592. doi:https://doi.org/10.1016/j.nima. 2020.163592

[13] C2 HV broadband amplifier, $2 \mathrm{GHz}, 40 \mathrm{~dB}$, https://cividec.at/ electronics-C2-HV.html (2020).

[14] Waverunner 8000, Teledynelecroy, http://cdn.teledynelecroy.com/ files/pdf/waverunner8000-datasheet.pdf (2018).

[15] S. Martoiu, et al., Development of the scalable readout system for micropattern gas detectors and other applications,, J. Instrum. 8 (2013) C03015. doi:http://dx.doi.org/10.1088/1748-0221/8/03/C03015.

[16] I. Manthos, et al. for the RD-51 PICOSEC Collaboration, Recent developments on precise timing with the picosec micromegas detector, Vol. 1498, Micro-Pattern Gaseous Detectors Conference 2019, Journal of Physics: Conference Series, 2020, p. 012014. doi:10.1088/1742-6596/1498/1/ 012014. 